\title{
Substantial tensile ductility in sputtered Zr-Ni-Al nano-sized metallic glass
}

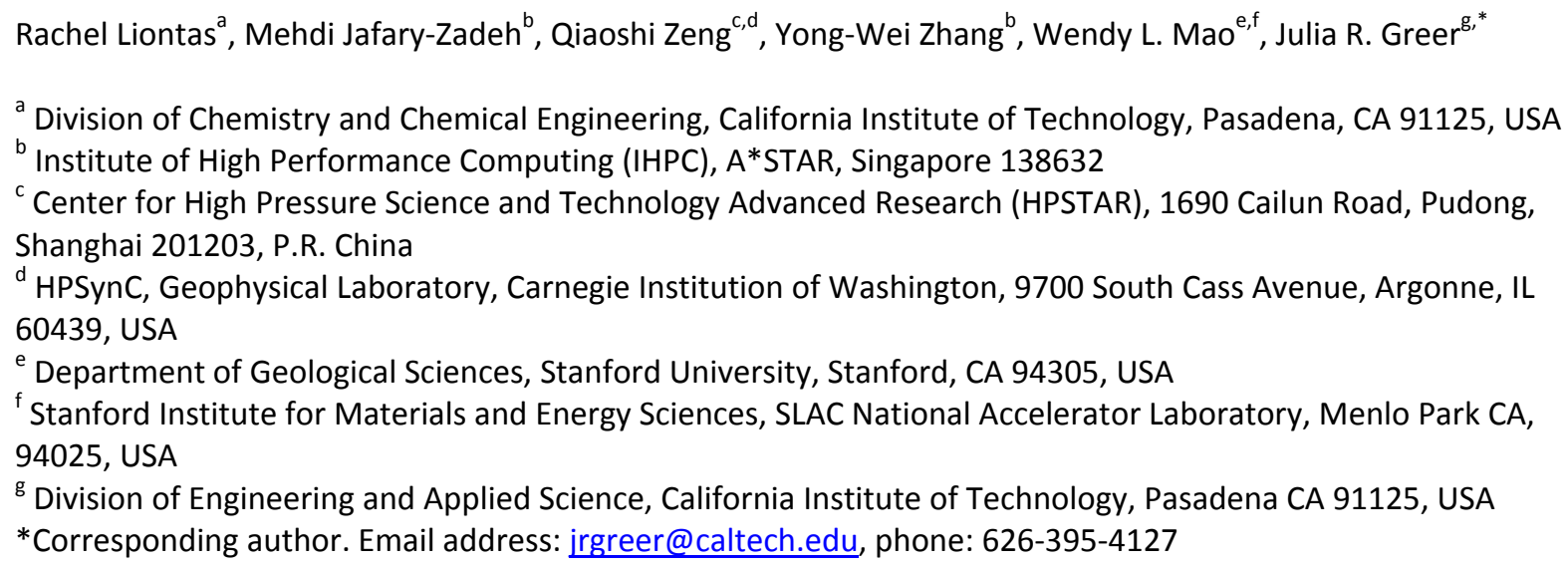

\section{Abstract}

We investigate the mechanical behavior and atomic-level structure of glassy $\mathrm{Zr}$-Ni-Al nano-tensile specimens with widths between 75 and $215 \mathrm{~nm}$. We focus our studies on two different energy states: (1) as-sputtered and (2) sputtered then annealed below the glass transition temperature $\left(T_{g}\right)$. In-situ tensile experiments conducted inside a scanning electron microscope (SEM) reveal substantial tensile ductility in some cases reaching $>10 \%$ engineering plastic strains, $>150 \%$ true plastic strains, and necking down to a point during tensile straining in specimens as wide as $150 \mathrm{~nm}$. We found the extent of ductility depends on both the specimen size and the annealing conditions. Using molecular dynamics (MD) simulations, transmission electron microscopy (TEM), and synchrotron $\mathrm{x}$-ray diffraction (XRD), we explain the observed mechanical behavior through changes in free volume as well as short- and medium- range atomic-level order that occur upon annealing. This work demonstrates the importance of carefully choosing the metallic glass fabrication method and post-processing conditions for achieving a certain atomic-level structure and free volume within the metallic glass, which then determine the overall mechanical response. An important implication is that sputter deposition may be a particularly promising technique for producing thin coatings of metallic glasses with significant ductility, due to the high level of disorder and excess free volume resulting from the sputtering process and to the suitability of sputtering for producing thin coatings that may exhibit enhanced size-induced ductility.

Keywords: metallic glass; ductility; in situ tension test; molecular dynamics; synchrotron XRD 


\section{Introduction}

Metallic glasses are a unique class of materials that possess an amorphous atomic-level structure, as in oxide glasses, and cohesive metallic bonding, as in metals. This combination of features leads to a host of desirable mechanical properties, including high strength and hardness, good wear and corrosion resistance, a large elastic strain limit, and low mechanical dissipation [1]. Room temperature deformation of metallic glasses typically involves localization of plastic strain into narrow bands, known as shear bands, resulting in catastrophic failure and a lack of tensile ductility [2]. Some monolithic bulk metallic glasses have been shown to exhibit significant plasticity under compression [3-7]; all exhibit virtually no extendibility $(<0.5 \%$ strain) under tension. Much recent research in metallic glasses has been dedicated to developing ways of accommodating plastic deformation to impart tensile ductility, to suppress catastrophic failure, and to improve the mechanical properties of metallic glasses, rendering them suitable in structural applications.

Tensile ductility at room temperature has been shown to emerge in monolithic metallic glasses only when the sample size is reduced to the nanoscale [8-10]. The extent of this tensile ductility was reported to vary widely as a function of the specific metallic glass and its processing conditions, with some studies indicating almost no plasticity (<1\%) even for sub-100 nm molded $\mathrm{Pt}_{57.5} \mathrm{Cu}_{14.7} \mathrm{Ni}_{5.3} \mathrm{P}_{22.5}[11,12]$ and electrodeposited $\mathrm{Ni}_{80} \mathrm{P}_{20}[13]$, others reporting strains of $23-45 \%$ for $100 \mathrm{~nm}$ copper-mold casted $\mathrm{Zr}_{52.5} \mathrm{Cu}_{17.9} \mathrm{Al}_{10} \mathrm{Ni}_{14.6} \mathrm{Ti}_{5}$ [8], $25 \%$ for $100 \mathrm{~nm}$ sized $\mathrm{Zr}_{35} \mathrm{Ti}_{30} \mathrm{Co}_{6} \mathrm{Be}_{39}$ [9], and others reporting elongation of $200 \%$ for $<20 \mathrm{~nm}$ nanohills of twin-jet electropolished $\mathrm{Al}_{90} \mathrm{Fe}_{5} \mathrm{Ce}_{5}$ [10]. The aim of this work is to understand the origins of such a wide range of post-elastic deformability in nano-sized metallic glasses, specifically focusing on the question of why certain nano-sized metallic glasses exhibit substantial ductility, in excess of $100 \%$ true strain.

For monolithic metallic glasses, the main research thrusts towards understanding why certain metallic glasses exhibit enhanced ductility have focused on the choice of alloying elements and the corresponding stoichiometry of those constituent elements. Some studies indicate that ductility can be brought about by choosing alloying elements such that the ratio of the elastic shear modulus to the bulk modulus is low, i.e. less than $0.41-0.43$ $[14,15]$. These investigations do not take into account the effects of the metallic glass fabrication method and processing conditions on ductility, which likely serve as key factors in governing post-elastic deformability in these materials. Metallic glasses may be formed through a variety of methods including splat quenching, melt spinning, 
copper-mold casting, arc melting, electrodeposition, and sputtering. A metallic glass formed by copper-mold casting could exhibit a significant difference in ductility than a metallic glass with the same chemical composition formed by sputtering. Even a single metallic glass fabrication method has some variability in processing conditions, such as the cooling rate of splat quenching, or the deposition conditions of sputtering, which could greatly influence the presence of free volume.

We investigated the mechanical behavior and atomic level structure of sputter-deposited $\mathrm{Zr}$-Ni-Al metallic glass. The mechanical response of this metallic glass was studied through in-situ uniaxial tension experiments conducted inside a scanning electron microscope (SEM) on nano-sized specimens with widths of 75-215 $\mathrm{nm}$. These nano-samples were fabricated by focused-ion beam (FIB) milling of sputtered Zr-Ni-Al in two different energy states: (1) as-sputtered and (2) sputtered and then annealed at $625 \mathrm{~K}$ for 24 hours. We chose $\mathrm{Zr}$-Ni-Al as our model metallic glass because of its oxidation resistance $[16,17]$ and excellent glass-forming ability $[18,19]$. The high $T_{g}$ of Zr-Ni-Al metallic glass, reported to range from 680 to $760 \mathrm{~K}$ depending on composition [20], increases the applicability of this metallic glass to extreme environments, such as those involving elevated temperatures and radiation, with a lower risk of crystallization or oxidation. The Zr-Ni-Al system contains only metallic components, which renders it a more representative metallic glass with true metallic bonding, as opposed to a system containing metalloid components with some covalent bonding. The properties of metal-metal systems are much better understood than those of metal-metalloid systems, whose covalent interatomic bonds influence glass formation and make modeling such systems difficult [21]. With only three component elements, this system is also practical for conducting molecular dynamics (MD) simulations. The shortage of literature on mechanical properties and detailed structural analysis of sputtered Zr-Ni-Al metallic glass motivated us to undertake a fundamental study of its mechanical behavior and atomic-level structure. The microstructure was analyzed by transmission electron microscopy (TEM) and synchrotron X-ray diffraction (XRD) to elucidate the unique atomic arrangement brought about by forming this metallic glass via sputtering and the changes in this structure that occur as a result of subsequent annealing. Mechanical behavior was assessed by conducting in-situ uniaxial tension and compression experiments: the tensile results are the focus of the current work, and the compression results are included in the Appendix Section A. MD simulations were conducted to explore the effect of metallic glass fabrication conditions, sub- $T_{g}$ annealing, and sample size. 


\section{Methods}

\subsection{Fabrication of free-standing Zr-Ni-Al nano-tensile specimens}

All samples in this study were fabricated from a sputtered $\mathrm{Zr}-\mathrm{Ni}$-Al metallic glass thin film. We deposit this material by sputtering an alloyed $\mathrm{Zr}_{56} \mathrm{Ni}_{22} \mathrm{Al}_{22}$ target ( $\mathrm{ACl}$ Alloys, Inc.) with a base pressure less than $1 \times 10^{-6}$ using a DC power supply at $100 \mathrm{~W}$ under 3 mTorr argon in a magnetron sputter deposition system (ATC Orion sputtering system, AJA International, Inc.). The sputtering process produces an amorphous $\mathrm{Zr}-\mathrm{Ni}$-Al film, which is the starting point for the fabrication of nano-tensile specimens. Samples were fabricated from two different amorphous states of this metallic glass: (1) as-sputtered and (2) sputtered then annealed at the sub- $T_{g}$ temperature of $625 \mathrm{~K}$ for 24 hours. For the as-sputtered state, specimens were fabricated by sputtering Zr-Ni-Al directly onto a silicon TEM grid (PELCO FIB Lift-Out TEM grid, Ted Pella) to a minimum thickness of $3 \mu \mathrm{m}$ as shown in Figure 1 Step (1-2) and then using $\mathrm{XeF}_{2}$ gas from the insulator enhanced etching system in a SEM to selectively etch away the silicon substrate underneath the sputtered Zr-Ni-Al. (Figure 1 Step (3)). In this step, a fine needle was positioned close to the sample surface to create a high local flux of $\mathrm{XeF}_{2}$ gas while the ion beam was used to induce reactions between the $\mathrm{XeF}_{2}$ gas and the scanned area. These efforts were to minimize and/or to eliminate any possible etching of the $\mathrm{Zr}$ - $\mathrm{Ni}-\mathrm{Al}$ metallic glass via the combined effect of the chemical selectivity of $\mathrm{XeF}_{2}$ for $\mathrm{Si}$ and by physically selecting only $\mathrm{Si}$ as the scanned area in the SEM. This resulted in the creation of a free-standing Zr-Ni-Al film directly on the TEM grid, which was then FIB-milled into the desired geometry of nano-tensile specimens shown in the insets of Figure 1 Step (4). The FIB-milling step in the specimen fabrication required extensive tilting and rotating of the SEM stage to achieve the desired removal of material. A schematic of this process is shown in Figure 1 . This methodology allowed fabrication of the specimens directly on the TEM grid, which facilitated the TEM analysis after tension experiments. The resultant specimens contained a test-section that was approximately square in cross-section with rounded corners. Specimens of this as-sputtered metallic glass were fabricated with widths ranging from $85-$ $215 \mathrm{~nm}$ and heights correspond to an aspect ratio of $\sim 1: 10$.

To fabricate specimens in the annealed state, the TEM grid with the $\sim 3 \mu \mathrm{m}$-thick sputter-deposited $\mathrm{Zr}$-Ni-Al film was annealed in a Carbolite tube furnace (in between Steps (2) and (3) of Figure 1). Prior to raising the temperature of the furnace, argon was flowed through the tube furnace at a rate of 3 liters per minute for 1 hour 
to avoid sample oxidation; this flow rate was maintained during the entire annealing process. The temperature of the tube furnace was increased from room temperature to the annealing temperature of $625 \mathrm{~K}$ at a rate of $45 \mathrm{~K}$ $\min ^{-1}$ and then held at the annealing temperature for 24 hours. From literature, the $T_{g}$ of $\mathrm{Zr}_{55} \mathrm{Ni}_{25} \mathrm{Al}_{20}$ is $757.1 \mathrm{~K}$ [20], which means the annealing temperature of $625 \mathrm{~K}$ roughly corresponds to $80 \%$ of $T_{g}$. After the 24 -hour annealing period, the furnace was turned off and allowed to reach room temperature before stopping the argon flow and removing the sample. Following annealing, nano-tensile specimens were fabricated using an identical FIBmilling process as the ones made from the as-sputtered state, with similar sizes of $79-209 \mathrm{~nm}$, and the aspect ratio of $\sim 1: 10$. Potential oxidation induced by the annealing was prevented by conducting the annealing in the argon environment. FIB-milling the specimens from a larger film of annealed Zr-Ni-Al metallic glass provided substantial oxidation protection as any surface oxide layers that may have formed during annealing were removed by the FIB, and the resultant specimen test-section consists of material at a distance greater than $1 \mu \mathrm{m}$ from the surfaces exposed to the annealing environment.

\subsection{TEM characterization}

TEM samples were prepared with the $\mathrm{Ga}^{+}$FIB following standard lamella preparation techniques and by fabricating the nano-tensile specimens directly onto the TEM grid (Figure 1). The FEI Technai TF20 transmission electron microscope was operated at $200 \mathrm{keV}$ to obtain bright field and dark field images, diffraction patterns, high-resolution TEM images, and to conduct energy dispersive X-ray spectroscopy (EDS) in STEM mode. To assess any changes in the atomic structure as a result of tension experiments, some nano-tensile specimens were left untested; the microstructure in both types of specimens - mechanically strained and as-fabricated - was examined by TEM. To analyze the overall sample composition and phase homogeneity, we fabricated the lamella lift-out sample from a $\mathrm{Zr}-\mathrm{Ni}-\mathrm{Al}$ film that had been sputtered for $\sim 20$ hours to a thickness of $\sim 4.5 \mu \mathrm{m}$. EDS was conducted at several points along the depth of the film to determine whether there were any changes in the chemical composition with sputter deposition time.

\subsection{In-situ uniaxial tension experiments}


Uniaxial tensile experiments were performed in-situ inside an SEM to allow simultaneous visualization of deformation and measurement of load and displacement during tensing. Tensile experiments were conducted using the Hysitron PI-85 nano-mechanical module in the FIB (FEI, Versa 3D). Tensile grips were fabricated by FIBmilling boron-doped diamond into a grip shape, shown in Figures 3 (a-d) and 4 (a-d). To perform a tension experiment, a nano-tensile specimen was moved inside the tension grips and then a displacement-controlled uniaxial tension experiment was conducted at a strain rate of $1 \times 10^{-3} \mathrm{~s}^{-1}$ until the point of failure. Loaddisplacement data was acquired at a rate of 200 data points per second; to display representative data points, the data was smoothed with a span containing data over the nearest $2 \mathrm{~nm}$ of the displacement, using the rlowess method in MATLAB, which involves a robust local regression using weighted linear least squares and a first degree polynomial model.

Load-displacement data was converted to stress and strain by measuring the relative dimensional changes throughout the experiments in each individual specimen. Engineering stresses and strains were calculated by utilizing the initial specimen height and cross-sectional area obtained from measurement of specimen height and width in SEM images taken prior to tensile experiments. True stresses and strains were calculated using the instantaneous specimen height and cross-sectional area during the tension experiment. To obtain these instantaneous specimen dimensions, we assumed that necking began at the maximum load during the tension experiment, and the criterion for necking was the presence of any stress-strain data beyond the maximum stress. We assumed that prior to necking the gauge length deformed uniformly, with $\sigma_{T}=\sigma_{E}\left(1+\varepsilon_{E}\right)$ and $\varepsilon_{T}=\ln (1+$ $\left.\varepsilon_{E}\right)$, where $\sigma_{T}$ is true stress, $\sigma_{E}$ is engineering stress, $\varepsilon_{E}$ is engineering strain, and $\varepsilon_{T}$ is true strain. To account for the large localization of deformation in the necked region that occurs upon necking, true stress-strain data was calculated after necking according to $\sigma_{T}=F / A_{\text {neck }}$ and $\varepsilon_{T}=\ln \left(\frac{A_{0}}{A_{\text {neck }}}\right)$ where $F$ is the load measured during the tension experiment, $A_{\text {neck }}$ is the cross-sectional area of the thinnest portion of the necked region, and $A_{0}$ is the initial cross-sectional area of the specimen. The width of the neck region $\left(w_{\text {neck }}\right)$ was measured at approximately 10 load-displacement points during necking. Due to the roughly square cross-sectional area of the specimens, the cross-sectional area of the necked region $\left(A_{\text {neck }}\right)$ was calculated from measured $\mathrm{w}_{\text {neck }}$ as $w_{\text {neck }}^{2}$ and $A_{\text {neck }}$ was assumed to vary linearly between each measurement point. 
Engineering stresses and strains were used to calculate the elastic modulus (E), yield strain $\left(\varepsilon_{y, \text { engineering }}\right)$, yield strength $\left(\sigma_{y}\right)$, engineering plastic strain $\left(\varepsilon_{p \text {, engineering }}\right)$, and ultimate tensile strength (UTS). The only mechanical property calculated form the true stress-strain data was the true plastic strain $\left(\varepsilon_{p, \text { true }}\right)$. The elastic modulus was calculated as the slope of the loading curve by conducting a linear fit on the region of the loading curve just after any initial loading instabilities and just before the yield point. The yield point was determined by visually inspecting the data for the first deviation from linear elastic loading and the yield stress and strain $\left(\sigma_{y}\right.$ and $\left.\varepsilon_{y, \text { engineering }}\right)$ were defined as the respective stress and strain values at this yield point. The UTS was found as the maximum value of stress in the engineering stress-strain response of a specimen.

\subsection{Synchrotron XRD Characterization}

Synchrotron XRD experiments were conducted on $\mathrm{Zr}-\mathrm{Ni}$-Al metallic glass in the same two states as the nanomechanical experiments: (1) as-sputtered and (2) sputtered then annealed at $625 \mathrm{~K}$. To obtain enough material for synchrotron XRD measurements, Zr-Ni-Al was deposited using identical sputter conditions discussed previously with kapton tape as the substrate, which allowed flakes of the sputtered $\mathrm{Zr}$-Ni-Al to be easily removed from the kapton tape and collected for analysis. Synchrotron XRD analysis was conducted on the as-sputtered Zr-Ni-Al flakes and annealed flakes, which were annealed prior to the diffraction measurements using identical annealing conditions discussed previously. Synchrotron XRD was performed in a transmission geometry using the highintensity, high-energy monochromatic beam at Sector 11-IDC of the Advanced Photon Source at Argonne National Laboratory. The photon energy was $105.1 \mathrm{keV}$ corresponding to an X-ray wavelength of $0.11798 \AA$ and the beam size was $0.5 \times 0.5 \mathrm{~mm}^{2}$. The X-ray attenuation length of the $\mathrm{Zr}_{55} \mathrm{Ni}_{25} \mathrm{Al}_{20}$ sample at $105.1 \mathrm{keV}$ is $\sim 2.5 \mathrm{~mm}$. $\mathrm{Zr}-\mathrm{Ni}-\mathrm{Al}$ flakes were sandwiched between two layers of kapton tape to increase the sample thickness (in this case $200 \mu \mathrm{m})$. A Perkin Elmer area detector was set at a distance of $690.7 \mathrm{~mm}$ and $\mathrm{CeO}_{2}$ was used as the calibration material. The exposure time was $90 \mathrm{~s}$ for each sample and the background scattering from air and the two layers of kapton tape. The resultant 2D image files were integrated using the Fit2D program to obtain 1D intensity distributions as a function of the wavevector $Q$, from which the structure factor $(S(Q))$ and the reduced pair-wise distribution function $(G(r))$ were derived using the PDFgetX3 program. The composition was assumed to be $\mathrm{Zr}_{55} \mathrm{Ni}_{25} \mathrm{Al}_{20}$ for data analysis. 


\subsection{Molecular dynamics simulations}

MD simulations were conducted on cylindrical Zr-Ni-Al metallic glass specimen using Large-Scale Atomic/Molecular Massively Parallel Simulator (LAMMPS) [22]. The interatomic interactions were calculated by employing the embedded atom model (EAM) potential parameterized for the $\mathrm{Zr}$-Ni-Al-Cu system [23]. To construct the samples, first, a $5 \mathrm{~nm}$ cube of the alloy with the atomic composition of $\mathrm{Zr}_{55} \mathrm{Ni}_{25} \mathrm{Al}_{20}$ (in the range of our experiments) and periodic boundary conditions (PBCs) along all three dimensions was melted and equilibrated at $2300 \mathrm{~K}$ for $10 \mathrm{~ns}$. Then, the liquid alloy was quenched to $50 \mathrm{~K}$ with a given cooling rate $\left(\right.$ e.g. $\left.10^{10} \mathrm{~K} / \mathrm{s}\right)$ at zero external pressure using the isothermal-isobaric (NPT ensemble) ensemble. To control the temperature and pressure of the simulations, Nose-Hoover thermostat and barostat were employed [24]. To integrate the equations of atomic motion, the Verlet algorithm [25] was used with the time step of 0.002 ps during the melting/quenching processes. The cylindrical sample for the uniaxial tensile test with diameter of $40 \mathrm{~nm}$ and length of $120 \mathrm{~nm}$ was then constructed by replicating the metallic glass cube. We applied PBCs in the direction parallel to the central axis of the cylinder (i.e. $z$ direction), while free boundary conditions were applied in other directions. Before applying the tensile loading, the sample was equilibrated at $300 \mathrm{~K}$ (similar to the temperature of our experiments). The uniaxial loading was applied by rescaling the simulation box in the $z$ direction with the strain rate of $4 \times 10^{7} \mathrm{~s}^{-1}$. During the loading procedure, the time step of the Verlet algorithm was set to $0.001 \mathrm{ps}$. The engineering stress was calculated from the Virial atomic stress tensor [26,27]. As a measure of local (atomistic) deformation during loading, we adopted the atomic von Mises strain [28] and used OVITO package for the visualization [29].

In the MD simulations, we examined the following three factors: cooling rate, annealing condition and sample size. To understand the effect of cooling rate (i.e. the effect of fabrication variables), we constructed the tensile samples using glasses obtained with cooling rates of $10^{10} \mathrm{~K} / \mathrm{s}, 10^{11} \mathrm{~K} / \mathrm{s}, 10^{12} \mathrm{~K} / \mathrm{s}$, and $10^{13} \mathrm{~K} / \mathrm{s}$. Moreover, to study the effect of sub- $T_{g}$ annealing (i.e. the effect of post-fabrication variables), the sample constructed with the cooling rate of $10^{13} \mathrm{~K} / \mathrm{s}$ was annealed for up to $400 \mathrm{~ns}$ at $850 \mathrm{~K}\left(\sim 0.8 T_{g}\right)$, and cooled back to $300 \mathrm{~K}$ with the same cooling rate. In addition, to study the size effect, tensile samples with diameters of $5,10,20$, and $40 \mathrm{~nm}$ and the same aspect ratio (i.e. ratio of length to diameter) of 3 were prepared using the glass obtained with the cooling rate of $10^{10} \mathrm{~K} / \mathrm{s}$. 
To characterize the short range order (SRO) present in the MD simulated samples we utilized the Voronoi tessellation method [30,31], whereby around each atom a Voronoi polyhedron is constructed with a certain number of triangular, quadrangular, pentagonal, and hexagonal faces, depending upon the arrangement of its nearest neighbor atoms. The Voronoi index of each atom corresponds to the number of faces in its surrounding Voronoi polyhedron, for example, a Voronoi index of $<0,2,8,1>$ corresponds to a Voronoi polyhedron with 0 triangular faces, 2 quadrangular faces, 8 pentagonal faces, and 1 hexagonal face. With this notation, a Voronoi index of $\langle 0,0,12,0\rangle$ corresponds to the Voronoi cell with the highest possible local symmetry, that is a regular dodecahedron with 12 nearest neighbor atoms arranged at the vertices of a full-icosahedron (F1) cluster. As an indicator of SRO, we mainly focus on tracking the concentration of the Al-centered full icosahedron cluster (i.e. AlFI with the Voronoi index of $\mathrm{Al}\langle 0,0,12,0\rangle)$ since this icosahedron configuration is known to be prevalent in a variety of metallic glass systems [32,33]. Other SRO clusters can also be tracked, as shown in Figure B.2 of the Appendix Section B. We chose to focus on the Al-centered icosahedron because we found this particular cluster to be most sensitive to changes in processing conditions, such as annealing and utilizing different cooling rates, as shown in Figure B.2 of the Appendix Section B. The average atomic potential energy of the system is another measure we utilized to assess the overall ordering in the system since a decrease in atomic potential energy signifies atoms in a more ordered, relaxed state.

\subsection{Virtues and limitations of molecular dynamics simulations in small-scale mechanics}

It is well-known that the spatial and temporal scales in MD simulations are very different from those in experimental techniques due to the restrictions in the technique and computational resources. The samples in current MD simulations typically contain up to tens of million atoms, which corresponds to a $\sim 40 \mathrm{~nm}$-diameter, $120 \mathrm{~nm}$-tall nano-pillar. The typical simulation duration (usually tens of nanoseconds), the cooling rate (as high as $10^{10} \mathrm{~K} / \mathrm{s}$ and above), and the strain rate (on the order of $10^{7} \mathrm{~s}^{-1}$ ) are several orders different than those of experiments. These limitations generally prevent a quantitative comparison between simulated and experimental results, which should not be the objective of MD simulations. For example, it has been shown that a higher strain rate in MD simulations often leads to a higher yield strength in materials [34]. Hence, to interpret simulation results, one should mainly focus on the major mechanistic features and trends in the behavior of the samples. If the simulation and experimental results follow the same trend, it would be plausible to use these simulations to 
reveal the underlying atomistic mechanisms, which are otherwise not obtainable via experimental techniques $[35,36]$. Based on these guiding principles, MD simulations have been extensively employed, together with experiments, to reveal valuable insight into complicated mechanical properties and behaviours, such as shear banding, crack initiation/propagation, and elastic buckling in a variety of metallic glass and nanoglass systems [3545].

\section{Results}

\subsection{TEM microstructure and elemental analysis}

TEM images and analysis of as-sputtered and annealed Zr-Ni-Al are presented in Figure 2. The film used for this analysis was sputtered for $\sim 20$ hours to a thickness of $\sim 4.5 \mu \mathrm{m}$. We confirmed this sample to be amorphous, evidenced by the presence of diffuse rings in the diffraction patterns (Figure 2(a)), as well as the lack of any observable ordering in the high-resolution TEM image (Figure 2(b)). Energy-dispersive x-ray spectroscopy (EDS) in the TEM was conducted on the cross-section of the as-sputtered film at various distances away from the substrate to determine the stoichiometric ratio of each component and to assess whether sputtering induced any variations in chemical composition with deposition time. The chemical composition based on the EDS data is plotted in Figure 2(c) and shows that chemical composition is not a function of distance away from the substrate, which indicates that the sputtering process is consistent over time. The horizontal lines shown in Figure 2(c) are plotted at the average composition for each element, with the legend displaying these average composition values with the standard deviation in that measurement. This plot implies that less than 2 at. \% of carbon and oxygen are present in the thin film lamella sample, with the average stoichiometry of $\mathrm{Zr} / \mathrm{Ni} / \mathrm{Al}$ being $\mathrm{Zr}_{55} \mathrm{Ni}_{29} \mathrm{Al}_{16}$.

The microstructure of annealed samples was analyzed by TEM to ensure that crystallization and oxidation had not occurred, these TEM results are shown in Figure 2(d)-(g). The TEM image of an entire nano-mechanical specimen (Figure 2(e)) reveals than an amorphous microstructure was retained upon annealing, as evidenced by the diffuse rings in the diffraction pattern (Figure 2(d)) and the uniform contrast dark-field image (Figure 2(f)). TEM analysis on post-tension samples indicated no evidence of crystallization upon mechanical deformation and no changes in the amorphous structure observable by TEM. As measured by EDS, the relative amounts of each element after annealing are shown in the chart in Figure 2(g) and reveal a slightly greater carbon and oxygen 
content compared with the as-sputtered sample (Figure $2(c)$ ) with both amounts $<10$ at. $\%$. This increased carbon and oxygen content stems from extensive SEM imaging during tension experiments and a small surface oxide layer from air exposure. The signal from these surface atoms is amplified for the nano-tensile specimen because of their high surface-to-volume ratio and the increased sensitivity of EDS to surface atoms. EDS analysis also revealed $\sim 2$ at. \% Ga present in the annealed specimens, most likely caused by the $\mathrm{Ga}^{+}$ions in the FIB, with a higher concentration of Ga near the sample surfaces where the ion beam came in direct contact with the sample to remove material, as shown in Figure C.1 of Appendix C. The amount of $\sim 2$ at. \% Ga is likely an upper bound for the actual amount of Ga present in the sample because EDS is more sensitive to surface atoms. The average stoichiometry of $\mathrm{Zr} / \mathrm{Ni} / \mathrm{Al}$ in the annealed nano-tensile specimens corresponds to $\mathrm{Zr}_{56} \mathrm{Ni}_{24} \mathrm{Al}_{20}$ (Figure $2(\mathrm{~g})$ ), which is slightly different than that of the as-sputtered film, which was $\mathrm{Zr}_{55} \mathrm{Ni}_{29} \mathrm{Al}_{16}$ (Figure 2(c)). This difference in stoichiometry may be caused by a slight preference for etching certain elements in the $\mathrm{XeF}_{2}$ and FIB etching steps. All specimens underwent the same etching procedure, so these effects should be consistent among all the nanotensile specimens.

\subsection{Nano-mechanical experiments}

Figures 3 and 4 display the tensile stress-strain response of the as-sputtered and annealed samples arranged by specimen dimensions and grouped into four width categories: $\sim 90 \mathrm{~nm}, \sim 110 \mathrm{~nm}, \sim 150 \mathrm{~nm}$, and $200 \mathrm{~nm}$. For each width category, Figures 3 and 4 each show three representative engineering stress-strain curves, one representative true stress-strain curve, and one representative SEM image of a tested specimen around the time of failure. We observed two distinct regimes of mechanical behavior in the as-sputtered: "small" specimens with width categories < $200 \mathrm{~nm}$ failed by pronounced localized necking and reached true strains in excess of $100 \%$. The "large" specimens in the $200 \mathrm{~nm}$ width category failed by shear banding and exhibited marginal ductility. The transition from localized necking (Figure $3(a-c)$ ) to shear banding (Figure $3(d)$ ) can be seen in the SEM images of the specimens around the time of failure; this transition is even more apparent from the in-situ videos of the tensile experiments. For example, the $212 \mathrm{~nm}$-wide as-sputtered sample (Video $1 \mathrm{~A}$ and 1B) fails catastrophically via shear banding, and the $114 \mathrm{~nm}$-wide as-sputtered sample (Video 2A and 2B) shows localized necking with the material drawing down to a point. 
The annealed specimens (Figure 4) also show two distinct regimes of mechanical behavior: the "small" specimen width category < 110 nm failed by noticeable localized necking and reached true strains around $100 \%$, "large" specimen width categories $\geq \sim 110 \mathrm{~nm}$ failed by shear banding with virtually non-existent ductility. The transition from localized necking (Figure $4(a)$ ) to shear banding (Figure $4(b-d)$ ) can be seen in the SEM images from the specimens around the time of failure. Analogous to the as-sputtered samples, the transition from localized necking to shear banding is unambiguous in the in-situ videos: the $209 \mathrm{~nm}$-wide annealed sample (Video $3 \mathrm{~A}$ and 3B) catastrophically shear bands at failure, the $109 \mathrm{~nm}$-wide annealed sample (Video 4A and 4B) shear bands after some post-elastic deformation, and the $93 \mathrm{~nm}$-wide annealed sample (Video $5 \mathrm{~A}$ and 5B) exhibits localized pronounced necking. We found the main difference between the as-sputtered and the annealed specimens to be the critical transition width at which the mechanical behavior switches from ductility with significant necking to shear banding. For the as-sputtered specimens, this transition occurred somewhere between 150 and $200 \mathrm{~nm}$, and for the annealed specimens, this transition occurred at around $100 \mathrm{~nm}$.

Beyond these two regimes of mechanical behavior, necking versus shear banding, we also observed differences within each regime of mechanical behavior as a function of specimen width. For example, the annealed specimens in width categories $\geq 110 \mathrm{~nm}$ all failed by shear banding, with an accompanying loss of ductility for larger and larger samples. As measured by engineering plastic strain, the $\sim 110 \mathrm{~nm}$ annealed specimens exhibited $3.2 \%$ more plasticity than the $\sim 150 \mathrm{~nm}$ annealed specimens, which exhibited 35\% more plasticity than the $\sim 200 \mathrm{~nm}$ annealed specimens. The shear-banding mechanism is not suppressed in samples of widths above the critical dimension but may require higher forces to initiate when specimen widths approach the critical dimension. A similar trend was observed in the necking regime for the as-sputtered specimens: all as-sputtered specimens in width categories < $200 \mathrm{~nm}$ failed by localized necking, with smaller specimens exhibiting more plasticity. For example, as measured by engineering plastic strain, the $\sim 110 \mathrm{~nm}$ as-sputtered specimens exhibited $45 \%$ more plasticity than the $150 \mathrm{~nm}$ as-sputtered specimens (Figure 3).

The summary of measured mechanical properties of the as-sputtered and annealed specimens is displayed in Figure 5. Again, we grouped the mechanical properties by specimen width into categories of $\sim 90 \mathrm{~nm}, \sim 110 \mathrm{~nm}$, $\sim 150 \mathrm{~nm}$, and $\sim 200 \mathrm{~nm}$. Some natural variation in specimen size for each category was caused by the FIB fabrication; this variation is represented by the horizontal error bars in Figure 5, which correspond to the standard 
deviation within each size grouping. The vertical error bars indicate the standard deviation in each measured mechanical property within each category of specimen width. We quantified the ductility in the specimens by measuring the plastic strain $\left(\varepsilon_{p}\right)$, defined as the total strain at fracture minus the strain at the yield point. Figure

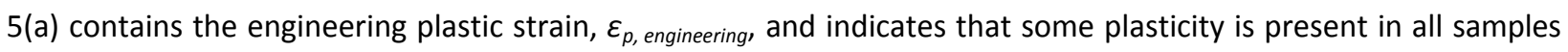
tested, with $\varepsilon_{p, \text { engineering }}$ ranging from $1.8 \%$ to $8.0 \%$. Figure $5(\mathrm{~b})$ contains the true plastic strain, $\varepsilon_{p, \text { true, which is }}$ sensitive to necking: the specimens with $\varepsilon_{p, \text { true }} \sim 100-150 \%$ all exhibited obvious necking, while specimens with $\varepsilon_{p \text {, }}$ true $<\sim 10 \%$ exhibited almost no necking with failure by shear banding.

Measures of strength are included in Figure 5(c) for yield strength, $\sigma_{y}$, and Figure 5(d) for ultimate tensile strength, UTS. It appears that the yield strength does not show a significant dependence on specimen size, but was highly influenced by annealing: the as-sputtered specimens exhibited on average $\sigma_{y}=1.26 \pm 0.08 \mathrm{GPa}$ and the annealed specimens exhibited on average $\sigma_{y}=1.51 \pm 0.11 \mathrm{GPa}$, which indicates that annealing resulted in a $\sim 20 \%$ increase in yield strength. The UTS appears to increase dramatically with annealing and with increasing the size of the annealed specimens, however since the UTS was calculated from engineering stresses, it is dramatically higher for samples that did not neck. Necking leads to a significant decrease in the cross-sectional area in the necked region, which means the sample is not able to accommodate total load as effectively as a sample that fails without such a localized narrowing. The UTS is commonly a more representative reflection of whether the samples undergo necking (low UTS) or fail by shear banding without necking (high UTS).

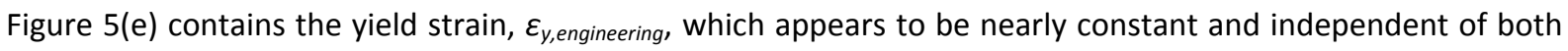
sample size and processing conditions. The average yield strain for all samples is $3.79 \pm 0.59 \%$. The consistency of the yield strain amongst all specimens indicates the elastic limit of this metallic glass is purely an intrinsic property that is not dependent upon specimen size, fabrication method (sputtering), or post-processing (annealing). As elastic modulus was measured as the slope of stress-strain curve up to the point of yielding, or simply the yield strength divided by the yield strain, this consistency in yield strain also indicates that any changes in the elastic modulus are due almost entirely to changes in yield strength, not yield strain. The elastic modulus measurements are shown in Figure 5(f) and are also roughly independent of specimen size, with the average modulus of $38.3 \pm$ 3.84 GPa for the as-sputtered specimens and $44.6 \pm 6.74 \mathrm{GPa}$ for the annealed specimens, which represents a $\sim 17 \%$ increase upon annealing. This increase is consistent with the $\sim 20 \%$ increase in yield strength of Figure 5 (c). A 
large standard deviation indicated by the error bars in the elastic modulus measurements is common in uniaxial tension experiments at the nanoscale because the modulus is measured from the slope of the loading portion of the nanomechanical data [46-48], which is susceptible to experimental artifacts like a slight misalignment between the grips and the sample, an imperfect initial contact, and the atomic-level roughness on the tensile grips and specimen head. The relative changes in the modulus are useful for showing the general trend of modulus increasing by $\sim 17 \%$ upon annealing; the absolute values of the measured moduli are likely lower than the actual moduli because of these artifacts.

\subsection{Synchrotron X-ray Diffraction}

To shed light onto the emergence of substantial ductility in excess of $100 \%$ true strain in the as-sputtered nanosized $\mathrm{Zr}$-Ni-Al metallic glasses and the dramatic changes in their mechanical response upon annealing, we conducted synchrotron XRD measurements on the as-sputtered and the annealed Zr-Ni-Al metallic glass samples. Figure 6(a) displays the resultant structure function $S(Q)$ as a function of the scattering vector $Q$, which contains noticeable changes in the first and second peaks between the as-sputtered and annealed samples. Figure 6(b) shows the zoomed-in first peak, which increases in intensity by $7.4 \%$ from $S\left(Q_{1}\right)=2.425$ in the as-sputtered sample to $S\left(Q_{1}\right)=2.604$ in the annealed sample and shifts by $1.7 \%$ from $Q_{1}=2.597 \AA^{-1}$ in the as-sputtered sample to $Q_{1}=2.641 \AA^{-1}$ in the annealed sample. $Q_{1}$ is inversely proportional to the mean atomic spacing [49], which implies that a larger $Q_{1}$ in the annealed sample corresponds to a smaller average atomic spacing compared with the assputtered sample. The position of $Q_{1}$ is related to the mean atomic volume; the variation in volume upon annealing can be estimated according to Yavari et al. [50] by,

$$
\frac{V_{\text {annealed }}}{V_{\text {as-sputtered }}}=\left(\frac{Q_{1}^{\text {as-sputtered }}}{Q_{1}^{\text {annealed }}}\right)^{3}
$$

where $V_{\text {annealed }}$ and $V_{\text {as-sputtered }}$ are the mean atomic volumes in the annealed stated and as-sputtered states,

respectively. Using equation (1) with the measured values $Q_{1}^{\text {as-sputtered }}=2.597 \AA$ and $Q_{1}^{\text {annealed }}=2.641 \AA$, we calculate a $\sim 4.9 \%$ lower atomic volume in the annealed sample compared with the as-sputtered sample, Indicative of a significant reduction in free volume due to annealing. Figure $6(\mathrm{c})$ contains the second peak in $S(Q)$, which increases in intensity by $\sim 1.6 \%$ from $S\left(Q_{2}\right)=1.210$ in the as-sputtered sample to $S\left(Q_{2}\right)=1.230$ in the annealed 
sample, as well as a peak split caused by the annealing giving rise to an additional peak at a $\sim 5.2 \AA^{-1}$, which reflects enhanced local ordering. The heights of the higher order peaks in $S(Q)$ decay rapidly as $Q$ increases, which render measurements of relative changes inaccurate.

Figure $6(\mathrm{~d})$ displays the reduced pair distribution function $G(r)$, defined as $G(r)=4 \pi r \rho_{0}(g(r)-1)$, where $r$ is the distance, $\rho_{0}$ is the average number density, and $g(r)$ is the pair distribution function. The pair distribution function $g(r)$ tends to 0 at small $r$ because atoms cannot overlap, which forces $G(r)$ to behave as $-4 \pi \rho_{0} r$ as $r \rightarrow 0$. At low $r$, the slope of $G(r)$ is proportional to the average number density of the material. We measured the slope of $G(r)$ for $r<2.25 \AA$ (Figure $6(\mathrm{e})$ ) and found it to increase by $7.2 \%$ as a result of the annealing, which corresponds to a $7.2 \%$ increase in density and a concomitant loss of free volume from annealing. Figure 6(f) contains a zoomed-in region of $G(r)$ focused on the peaks corresponding to the first nearest neighbor shell of atoms. These peaks appear to undergoes significant changes from ex-situ annealing and are important in understanding changes in shortrange order. In the as-sputtered state, the main peak at $\sim 3 \AA$ is split into two maxima: one sub-peak at $~ 2.7 \AA$ and another sub-peak at $3.2 \AA$. Upon annealing, the first sub-peak remains at $\sim 2.7 \AA$ and increases in intensity by $37.3 \%$ from $G(r)=0.984$ in the as-sputtered state to $G(r)=1.351$ in the ex-situ annealed state, while the second subpeak at $\sim 3.2 \AA$ is largely unchanged, increasing marginally from $G(r)=2.089$ in the as-sputtered state to $G(r)=2.098$ in the annealed state. This indicates a change in the types of atoms within the nearest neighbor shell, with ones whose bonds of length $\sim 2.7 \AA$ becoming more prevalent and those with $\sim 3.2 \AA$ bond length not changing significantly. Based on the atomic radii of each constituent in the $\mathrm{Zr}$-Ni-Al metallic glass, metallic bond lengths can be calculated [51], with results shown in Table 1, and denoted by the dashed lines in Figure 6(f). This plot reveals that upon annealing, the $\mathrm{Ni}-\mathrm{Al}$ and $\mathrm{Zr}$ - Zr bonds become more prevalent because the first sub-peak located at around the $\mathrm{Ni}-\mathrm{Al}$ bond distance intensifies and the second sub-peak shift closer towards the $\mathrm{Zr}$ - $\mathrm{Zr}$ bond distance. $\mathrm{Zr}$ atoms represent the majority of all atoms in the $\mathrm{Zr}$-Ni-Al glass in this work, with $\mathrm{Ni}$ and Al being the minority atoms; the observed changes in bond prevalence suggest that upon annealing the minority atoms ( $\mathrm{Ni}$ and $\mathrm{Al}$ ) come together significantly while the majority atoms $(\mathrm{Zr})$ also come together slightly. This leads the atomic-level structure in the annealed state to contain more minority-minority ( $\mathrm{Ni}-\mathrm{Al}$ ) and majority-majority ( $\mathrm{Zr}$ - $\mathrm{Zr}$ ) atom interactions but less majority-minority ( $\mathrm{Zr}-\mathrm{Al}$ or $\mathrm{Zr}$-Ni) atom interactions. 


\begin{tabular}{c|c} 
Bond & Length (Å) \\
\hline $\mathrm{Ni}-\mathrm{Ni}$ & 2.492 \\
\hline $\mathrm{Ni}-\mathrm{Al}$ & 2.678 \\
\hline $\mathrm{Zr}-\mathrm{Ni}$ & 2.848 \\
\hline $\mathrm{Al}-\mathrm{Al}$ & 2.864 \\
\hline $\mathrm{Al}-\mathrm{Zr}$ & 3.034 \\
\hline $\mathrm{Zr}-\mathrm{Zr}$ & 3.204
\end{tabular}

Table 1: Metallic bond lengths, obtained from published metallic bond radii in reference [51]

The peaks in $G(r)$ located beyond the first main peak also change considerably as a result of annealing. Table 2 summarizes the peak positions of these medium-range order (MRO) peaks in the as-sputtered and annealed sample and shows that (1) all peaks shift to shorter distances (by 1\%) consistent with an overall densification, and (2) the intensity increases by $18-48 \%$, which corresponds to more atoms at certain set radial distances and fewer atoms distributed randomly. These measurements imply that annealing leads to a dramatic increase in coordination number and medium-range order.

\begin{tabular}{c|c|c|c|c|c}
\multicolumn{3}{c|}{ Peak Location in G(r) } & \multicolumn{3}{c}{ Peak Intensity in G(r) } \\
\hline As-sputtered & Annealed & Change & As-sputtered & Annealed & Change \\
\hline 5.24 & 5.19 & $-0.95 \%$ & 0.669 & 0.787 & $17.7 \%$ \\
\hline 7.64 & 7.59 & $-0.65 \%$ & 0.468 & 0.605 & $29.2 \%$ \\
\hline 10.2 & 10.1 & $-1.31 \%$ & 0.209 & 0.269 & $29.2 \%$ \\
\hline 12.6 & 12.4 & $-1.04 \%$ & 0.107 & 0.158 & $48.1 \%$ \\
& & \multicolumn{2}{c}{ Table 2: Analysis of the MRO peaks in G(r) }
\end{tabular}

\subsection{Molecular dynamics simulations}

We employed molecular dynamics (MD) simulations to gain insight into the mechanisms of annealing-induced atomic ordering and structural relaxation revealed by the synchrotron XRD measurements. Due to lack of longrange order in metallic glasses, their atomic structure can only be characterized in a statistical manner. Indeed, the structure factor and pair distribution function obtained experimentally are one-dimensional entities indicating the possibility of finding another atom at a distance $r$ from a given central atom. In contrast, MD simulations are able to reproduce the atomistic configuration of metallic glasses and characterize their three-dimensional structure, which is not otherwise possible experimentally. Hence, molecular dynamics is almost a unique technique in exploring the correlation between microstructure, atomic-level details, and mechanical behavior of metallic glasses 
under different conditions. Here, we investigated the effect of structural relaxation on the deformation and failure mode of metallic glasses by adjusting the sample cooling rate, and the specific contributions of thermal energy by adjusting the annealing time at a sub- $T_{g}$ temperature. We also investigated the size effect by performing simulations on samples with different diameters (details of our simulations and sample preparation are provided in the Methods section).

Figure $7(\mathrm{a})$ shows the engineering stress-strain curves at room temperature $(300 \mathrm{~K})$ for the glassy specimens obtained with different cooling rates of $10^{10} \mathrm{~K} / \mathrm{s}$ to $10^{13} \mathrm{~K} / \mathrm{s}$, in order of magnitude increments. For each of these samples, Figure $7(b-e)$ present the snapshots of atomic von Mises shear strain, $\varepsilon^{v M}$, calculated at different stages of the applied strain, $\varepsilon$, with respect to the unloaded sample. The loading stage of each snapshot is indicated by an open circle in the stress-strain curve of the corresponding cooling rate in Fig 7(a). According to Figure $7(a)$, in the sample with the cooling rate of $10^{10} \mathrm{~K} / \mathrm{s}$, exhibits a pronounced drop in the stress level after the ultimate tensile strength (UTS); this can be attributed to formation and propagation of a dominant shear band as seen in Figure 7(b), which implies a brittle failure mode at room temperature in the glassy sample obtained with the slowest cooling. In contrast, Figure $7(\mathrm{a})$ also demonstrates that increasing the cooling rate above $10^{10} \mathrm{~K} / \mathrm{s}$ leads to an altering of the deformation mode toward more ductile (plastic) behavior and almost an ideally plastic flow in the sample formed with the cooling rate of $10^{13} \mathrm{~K} / \mathrm{s}$. This is in agreement with Figure $7(\mathrm{c}-\mathrm{e})$, indicating a homogeneous distribution of atomic deformation in these samples. Hence, Figure 7(a-e) clearly shows a transition in the roomtemperature deformation and failure mode from localized shear banding (in the sample formed with the slowest cooling rate) to homogeneous plastic flow (in samples formed with faster cooling rates).

We further consider the effect of cooling rate on atomic structure by investigating the SRO clusters and average potential energy per atom. Figure $7(f)$ demonstrates that a faster cooling rate leads to a more disordered atomic structure as evidenced by a decrease in the fraction of Al-FI clusters and by an increase in the average atomic potential energy of the system at room temperature. In other words, increasing the cooling rate results in a glass with a less "relaxed" structure consisting of more "liquid-like" clusters (i.e. excess free volume with irreversible dynamics). This reduction in full-icosahedra clusters at faster cooling rates corresponds to a destruction of the stiff backbone of the glass, which may be a key factor responsible for the homogeneous plastic deformation observed in samples formed at higher cooling rates. 
We performed further simulations to investigate the effect of sub- $T_{g}$ annealing on the structure and mechanical properties of the metallic glass. To this end, we selected the sample obtained with the cooling rate of $10^{13} \mathrm{~K} / \mathrm{s}$, whose tensile deformation commenced in a homogeneous fashion (see Figure $7(\mathrm{a}, \mathrm{e})$ ). The annealing temperature in the simulation was selected as $850 \mathrm{~K}$, which is $~ 80 \%$ of the MD-determined $T_{g}$ in this glass. We chose this annealing temperature to be consistent with the experimental annealing conditions performed at $\sim 80 \%$ of the experimental $T_{g}$ taken from literature [20]. The $T_{g}$ of the MD-simulated system was determined by considering the variation in the average atomic potential energy as a function of temperature during quenching and finding the temperature at which there was a change in the slope, as shown in Figure B.2 of Appendix B. The annealing simulation was conducted for up to $400 \mathrm{~ns}$; the average potential energy per atom and the fraction of AlFl clusters were monitored during the annealing process. Figure 8 illustrates the effect of annealing on the mechanical properties and atomic-level structure of simulated $\mathrm{Zr}$-Ni-Al glass. We observe that annealing the sample that was originally formed at a cooling rate of $10^{13} \mathrm{~K} / \mathrm{s}$ leads to a $77 \%$ increase in the ultimate tensile strength after 200 ns of annealing time (Figure 8(a)) and shifts the mechanical deformation from homogeneous extension of the as-quenched sample to brittle behavior and shear banding, as illustrated in Figure 8(b)-(c). Figure $8\left(\right.$ a) also includes the stress-strain responses of the as-quenched samples originally formed at cooling rates of $10^{13}$ and $10^{10} \mathrm{~K} / \mathrm{s}$, which are provided to show that annealing the sample originally quenched at a cooling rate of $10^{13}$ $\mathrm{K} / \mathrm{s}$ leads to a stress-strain response similar to that of the as-quenched sample formed at a cooling rate of $10^{10} \mathrm{~K} / \mathrm{s}$. Figure 8(d) shows a plot of potential energy per atom and percentage of Al atoms centered in full icosahedron, i.e. Al- $\mathrm{Fl}$, versus annealing time. This figure illustrates that increasing annealing time from $0 \mathrm{~ns}$ to $400 \mathrm{~ns}$ brings about atomic ordering of the system, as evidenced by a $0.2 \%(\sim 0.01 \mathrm{eV})$ decrease in the potential energy per atom and a $38 \%$ increase in the fraction of $\mathrm{Al}$ atoms located in the central position of full-icosahedra clusters. These results support that slower cooling rates and greater annealing times lead to the creation of samples with more relaxed and well-ordered microstructures, with a stronger and more brittle mechanical response, than that of samples with more disordered structures obtained by cooling at faster rates, which exhibit more ductile responses.

Previous studies have shown that the structural relaxation during sub- $\mathrm{T}_{\mathrm{g}}$ annealing is mainly attributed to localized atomic rearrangements (i.e. $\beta$-relaxation), which are manifested in processes such as enhancement in short-range ordering and/or annihilation of free volume [52,53]. In general, the temperature dependence of $\beta$ - 
relaxation follows the Arrhenius relation [53]. Previous experimental and theoretical studies suggested that the activation energy of such processes is on the order of 0.1 to $1 \mathrm{eV}$ [54-56]. Hence, the estimated relaxation time for such processes at $0.8 \mathrm{~T}_{\mathrm{g}}$ is on the order of sub-nanosecond to tens of nanoseconds. This implies that the $400 \mathrm{~ns}$ time used in our annealing simulations at $0.8 \mathrm{~T}_{\mathrm{g}}$ should allow ample structural relaxations of the metallic glass samples through localized $\beta$-relaxation. This is the primary reason why there is the same trend in the strengthening and embrittlement between our simulation results and experiment results.

Figure 9 illustrates how sample size can have a significant influence on mechanical response. In this figure, MD simulations of tensing different-diameter nano-cylinders reveal that atomic von Mises strains localize into an obvious shear band in the $40 \mathrm{~nm}$ diameter sample (Figure 9(b)). As the sample diameter is reduced to $20 \mathrm{~nm}$ the deformation becomes less localized but still exhibits shear banding (Figure 9(c)), decreasing the sample diameter to $10 \mathrm{~nm}$ changes the failure mechanism to necking (Figure 9(d)), and reducing the sample size to $5 \mathrm{~nm}$ leads to even more pronounced necking (Figure 9(e)). Figure 9(a) shows the simulated engineering stress-strain data and also demonstrates the shift in failure mechanism as the 40- and 20-nm diameter samples exhibit a sudden drop in stress after yielding due to shear band formation, while the 10- and 5-nm diameter samples exhibit gradual decreases in stress after yielding due to the gradual decrease in cross-sectional area of the necked region. The substantial fluctuations in the stress-strain response of the 5 and $10 \mathrm{~nm}$ samples after yielding is likely caused by the stronger effect of atomistic fluctuations in the small samples after each localized atomic yielding event.

\section{Discussion}

\subsection{Structural Relaxation Effects}

The ductility observed in the as-sputtered metallic glass specimens is substantial, attaining true strains of $\sim 150 \%$ and exhibiting visible tensile necking down to a point even in specimens as large as $\sim 150 \mathrm{~nm}$. This represents a significant departure from findings in previous studies, see for example $[8,9,12,13]$ where nanotensile metallic glass samples were formed by a variety of other non-sputtering processes. This departure may be attributed to the relatively unexplored effect of the sputtering process on the microstructure of the formed metallic glass. Previous studies on small-scale tension of metallic glasses nanopillars have utilized various nonsputtering fabrication methods, including thermoplastic moulding which led to almost no plasticity even for sub- 
$100 \mathrm{~nm} \mathrm{Pt} t_{57.5} \mathrm{Cu}_{14.7} \mathrm{Ni}_{5.3} \mathrm{P}_{22.5}$ specimens [12], electrodeposition which led to plastic strains of $2 \%$ in $\sim 100 \mathrm{~nm} \mathrm{Ni}_{80} \mathrm{P}_{20}$ specimens [13], and copper-mold casting which led to plastic strains of $23-45 \%$ for $100 \mathrm{~nm} \mathrm{Zr_{52.5 }} \mathrm{Cu}_{17.9} \mathrm{Al}_{10} \mathrm{Ni}_{14.6} \mathrm{Ti}_{5}$ specimens [8]. One study on the mechanical properties of small-scale sputtered metallic glass was conducted on $\mathrm{Pd}_{77} \mathrm{Si}_{23}$ metallic glass under compression, which revealed a suppression of failure localization into catastrophic shear bands for specimens with diameters up to $400 \mathrm{~nm}$ [57]. This $400 \mathrm{~nm}$ diameter is substantially larger than typical diameters reported for the size-induced brittle-to-ductile transition, on the order of $100 \mathrm{~nm}$, and is consistent with the compressive deformability observed in our $\mathrm{Zr}$-Ni-Al samples with diameters up to $555 \mathrm{~nm}$. A full discussion on these compression results is provided in Appendix Section A. This suggests that sputtering may be a method of metallic glass fabrication to create ductile metallic glasses of a variety of chemical compositions.

The results of this work reveal that the observed substantial ductility in the as-sputtered nano-sized Zr-Ni-Al stems from the sputtering process creating a metallic glass was in a substantially unrelaxed structural configuration with a large amount of residual free volume (i.e. liquid like atomic clusters with irreversible dynamics) in the vitrified state. Annealing these sputtered metallic glasses increased the atomic mobility and induced a relaxation process with structural rearrangements that annihilate some excess free volume, according to free volume theory [58]. This work demonstrates that the original metallic glass fabrication method i.e. sputtering, and post-processing i.e. annealing, dramatically affect the atomic-level structure, and hence, mechanical behavior, and failure mode in small-scale metallic glasses. This suggests that the design of systems containing metallic glasses must carefully consider the desired mechanical properties and select the fabrication method, the chosen size, and the post-processing conditions accordingly. The annealing in the current work was done for a long time period of 24 hours. It is likely that more moderate annealing would allow some of the benefits from annealing such as increased strength while maintaining significant ductility. The two systems of the current work: (1) as-sputtered and (2) annealed for 24 hours at $625 \mathrm{~K}$, represent two extremes of possible behavior. By exploring and adjusting the annealing and sputtering conditions, as well as the sample dimensions, it may be possible to obtain metallic glass with specifically desired mechanical properties, in a "materials by design" paradigm.

\subsection{Size Effects}


Both nano-tensile experiments and simulations revealed a consistent trend in the transition from shear banding to necking with reduced sample diameter: in experiments, this transition occurred between 150 and 200 $\mathrm{nm}$ for as-sputtered samples and at $\sim 100 \mathrm{~nm}$ for annealed samples, in simulations the transition occurred between 10 and $20 \mathrm{~nm}$. The smaller diameter necessary for the brittle-to-ductile transition in simulations is in agreement with several other MD studies of size effects in nano-sized metallic glasses $[59,60]$ and is commonly attributed to the substantially higher strain rates used in the MD simulations $\left(4 \times 10^{7} \mathrm{~s}^{-1}\right.$ in $\mathrm{MD}$ compared to $1 \times 10^{-3} \mathrm{~s}^{-1}$ in experiments). Faster strain rates have been associated with an increased propensity towards shear banding and a loss of ductility $[34,61,62]$. Besides, this smaller diameter for the brittle-to-ductile transition in simulation has also been attributed to the lack of surface imperfections such as surface roughness in simulated metallic glass samples as compared to experimental samples [26].

The size-induced ductility may be explained, in part, by surface effects: as the sample becomes smaller, the effect of the surface becomes more significant, as reported in the work of Chen et al. [13]. Surface atoms contain broken bonds and thus cannot form clusters with high local symmetries, such as full icosahedra, which means there is zero concentration of full icosahedra on surfaces and surfaces are structurally liquid. As the sample becomes smaller, the fraction of total atoms in this highly disordered surface state becomes larger, which means their contribution to mechanical behavior also becomes larger. The novelty of this work is that our results provide a direct link between the disorder in the atomic-level structure, quantified by synchrotron XRD experiments, and the commencement of deformation and failure, as well as confirmation that a larger fraction of atoms in the highly disordered state of surface atoms contributes to the ubiquitously observed emergent ductility in nano-sized metallic glasses.

The surfaces of the metallic glass specimens in this study may also be important in explaining the sizeinduced ductility because the specimens were fabricated using $\mathrm{Ga}^{+}$ion irradiation from a FIB. The influences of this $\mathrm{Ga}^{+}$ion irradiation on small-scale-specimens have been thoroughly explored in the last decade [63-65]. In particular, ion beam irradiation of metallic glasses has been shown to introduce defects, increase free volume, and cause the growth of nanocrystallites in the amorphous matrix [66-68]. We did not observe any nanocrystallites in the TEM analysis of the $\mathrm{Zr}$-Ni-Al nanomechanical specimens; it is difficult to accurately assess defects in amorphous materials or changes in free volume with TEM. According to Monte Carlo based simulations conducted with The 
Stopping and Range of lons in Matter (SRIM) software, glancing angle irradiation of $\mathrm{Zr}_{55} \mathrm{Ni}_{25} \mathrm{Al}_{20}$ with an assumed bulk density of $6.33 \mathrm{~g} \mathrm{~cm}^{-3}$ by $30 \mathrm{keV} \mathrm{Ga}^{+}$ions results in $78 \%$ of the implanted Ga present within the first $10 \mathrm{~nm}$ of the free surface of the specimen, and $\sim 98 \%$ of the implanted Ga present within the first $20 \mathrm{~nm}$ of the free surface of the specimen. This implies that the majority of implanted Ga is located very close to the surfaces of specimens. The detailed plot of Ga ion penetration depths calculated by SRIM is included in the Appendix Figure C.1. It is possible that the presence of Ga near the surfaces and Ga bombardments from FIB may influence the mechanical response of nano-sized metallic glasses, which have been shown to exhibit enhanced ductility and a slight suppression of shear banding upon FIB-induced Ga-ion irradiation [11-13]. This effect would be more pronounced for smaller specimens, which would have a correspondingly larger fraction of the specimen exposed to FIB; therefore FIB processing may be a contributing factor in the observed size effect. However because samples of the same size were fabricated with identical FIB conditions, the differences between the as-sputtered and annealed sample sets are likely not due to FIB effects. As discussed in section 2.1, the annealing was performed from a larger $\sim 3 \mu \mathrm{m}$ thick film of material prior to any FIB exposure and then after annealing the FIB was used to fabricated specimens. This methodology prevented any possible annealing-induced changes to the resultant FIB damage. This makes a direct comparison between Figure 3 (samples FIB-fabricated from the as-sputtered film) and Figure 4 (samples FIB-fabricated from the annealed film) justified. The large difference in ductility between the samples shown in Figures 3 and 4 are due to differences from the samples being in an as-sputtered or annealed state, and likely cannot be attributed to FIB processing. Nevertheless, it should be noted that as all specimens in this study were FIB-fabricated, there is no reference state without FIB exposure. Therefore, even the differences between specimens could be influenced by the disorder introduced by the FIB and the limits of disorder allowed by the material. The possible effects of FIB do not significantly influence the takeaway message of this work, which is to demonstrate the comparison between the as-sputtered and annealed small-scale metallic glass specimens. This comparison illustrates the annealing leads to an increase in atomic-level ordering and loss of free volume, which leads to a loss of ductility. In this work, we are less interested in the absolute mechanical response and are focused on the relative comparison between the as-sputtered and the annealed specimens, which experienced identical FIB exposure. 
Several previous studies reported a range of explanations for the dependence of plastic deformation on sample size. For example, Chen et al. and Volkert et al. [13,57] have rationalized the observed size effect in terms of energetics and postulated that for a sample with a characteristic dimension $d$, the total elastic strain energy stored in a sample scales with the sample volume or $\sim d^{3}$, while the surface energy that a shear band has to overcome to propagate scales with the cross-sectional area of the sample or $\sim d^{2}$. Therefore below a critical diameter, the elastic energy $\left(\sim d^{3}\right)$ is less than the shear banding energy $\left(\sim d^{2}\right)$ and it is energetically unfavorable for a shear band to form and propagate. Another reason for size-induced ductility is that the ductile specimens in this study may be smaller than the critical size of a shear band. One study suggested that for a shear band to fully develop and propagate, its nucleus would have to be $\sim 50-500 \mathrm{~nm}$ [2] and another study suggested that embryonic shear bands, this is shear bands prior to their catastrophic propagation, require a certain running distance in order to become critical [8]. Small specimens, like those studied in this work, may not provide a sufficient nucleation zone or running distance to allow for propagation of shear bands. Others have attributed the observations of size-induced ductility in metallic glasses to a competition between the energy required for crack-like shear band propagation and that for homogeneous flow [9,57]. Volkert et al. and Jang et al. argue that analogous to Griffith's criterion for crackpropagation, the critical stress necessary to propagate a pre-existing crack-like shear band is $[9,57,69]: \sigma=\sqrt{\frac{2^{3 / 2} \Gamma E}{a d}}$ where $\Gamma$ is the shear band energy per unit area, $E$ is the elastic modulus, $a$ is the aspect ratio (height/diameter) of the sample, and $d$ is the sample diameter. Thus the critical stress necessary to propagate a shear band decreases with increasing sample size. In larger samples, this critical stress is less than the stress for driving homogeneous flow, so shear bands are inevitable. In smaller samples, like those of the current work, the critical stress for shear bands is greater than the stress for homogeneous flow, so it is favorable for many shear transformation zones to activate throughout the sample, resulting in more homogeneous deformation and ductility $[9,57]$.

In the context of the described existing state of the art knowledge on deformation and energetics of nano-sized metallic glasses, our work demonstrates that the critical size for the emergence of ductility is not a fixed value for a given metallic glass; it is a strong function of the free volume distribution and the atomic-level energy state. By adjusting the fabrication method and post-processing steps, it is possible to push this critical size to induce ductility to even larger length scales. The parameters yet to be explored include changing the metallic glass composition 
and modifying the sputtering conditions, like power, argon gas pressure and flow rate, RF versus DC sputtering, cosputtering $\mathrm{Zr}, \mathrm{Ni}$, and $\mathrm{Al}$ instead of sputtering from an alloyed target, etc. Optimizing these parameters along with others that have been shown to increase ductility, such as ion irradiation $[12,70]$ and utilizing chemistries with a low ratio of the elastic shear modulus to the bulk modulus [14,15], may allow for the formation of a grossly disordered metallic glass with exceptional ductility. We were able to achieve unique properties in nano-sized metallic glasses by increasing their atomic-level disorder via sputter deposition; metallic glasses were originally discovered by rapidly quenching metallic alloys to suppress crystallization and bring about a disordered arrangement of atoms. Forming metallic glasses by sputtering allowed us to increase the effective quenching rate and to achieve one of the highest-yet reported ductility in small-scale metallic glasses, which sets them even further apart from crystalline metals and alloys. Controlling the atomic-level disorder enables us to elicit new mechanical properties, such as enhanced ductility, while maintaining the high strength and elasticity intrinsic to metallic glasses.

\section{Conclusions}

In-situ tensile experiments conducted inside a SEM demonstrate substantial ductility in nano-sized sputtered Zr-Ni-Al metallic glasses. The observed ductility is larger than that reported for other metallic glasses, reaching $>10 \%$ engineering plastic strains, $>150 \%$ true plastic strains, and necking down to a point during tensile straining in relatively large samples, of widths up to $150 \mathrm{~nm}$. The ductility was highly dependent on specimen size and annealing conditions, with the greatest ductility of $\sim 150 \%$ true strain observed in $\sim 90$ nm wide as-sputtered specimens, and all annealed specimens of equivalent dimensions were substantially less ductile. The combinations of molecular dynamics simulations, TEM microstructural analysis, and synchrotron XRD characterization techniques were used in concert to assess and to explain the observed mechanical behavior in the framework of free volume reduction and a concomitant increase in short- and medium- range order that occurred upon annealing. These findings illustrate the key role that the presence and distribution of free volume plays in governing the mechanical deformation, and in particular, ductility.

This work demonstrates the importance of not only the sample dimension but also the original metallic glass fabrication method (sputtering) and post-processing conditions (annealing) in tuning the mechanical properties of 
a metallic glass with a desired set of properties - potentially for a chosen application. The emergence of tensile ductility and necking in the as-sputtered $\mathrm{Zr}$-Ni-Al with widths up to $\sim 150 \mathrm{~nm}$ points towards sputtering as a promising technique for fabricating metallic glasses, particularly as thin coatings. Sputtering is well suited for deposition of thin coatings, and this work shows that the thinness of a sputtered metallic glass coating has the additional benefit of enhanced size-induced ductility that can be observed at relatively large critical dimensions, such as $\sim 150 \mathrm{~nm}$ shown in this work. Future work will be aimed at optimizing the sputtering parameters and other conditions that have been shown to influence ductility, such as utilizing systems containing low ratio of the elastic shear modulus to the bulk modulus $[14,15]$ and post-processing by ion irradiation $[12,70]$. Such a thorough exploration of parameter space will lead to developing processing techniques for the creation of optimally disordered metallic glasses with maximized ductility at even larger critical dimensions. Creating such ductile metallic glasses at larger length scales would increase the feasibility of utilizing metallic glasses in structural and technological applications, which require not only the high strength, elasticity, and corrosion resistance intrinsic to metallic glasses, but also the tensile ductility, which has been very difficult to achieve.

\section{Acknowledgements}

The authors gratefully acknowledge financial support from the National Science Foundation through R.L.'s Graduate Research Fellowship under grant DGE-1144469, the U.S. Department of Energy (DOE) through J.R.G.'s Early Career Research Program under grant DE-SC0006599, NASA's Space Technology Research Grants Program through J.R.G.'s Early Career Faculty Grant, and the National Academies Keck Futures Initiative under grant NAKFI ANT1. The authors thank Jonathan Thompson for help with annealing using the Carbolite tube furnace in the Lewis Group at Caltech, David Chen for helpful discussions, and the Atwater Group at Caltech for use of the sputter deposition system. The authors also thank the Kavli Nanoscience Institute (KNI) at Caltech for support and availability of cleanroom facilities. The authors also gratefully acknowledge the financial support from the Agency for Science, Technology and Research (A*STAR), Singapore and the use of computing resources at the A*STAR Computational Resource Centre, Singapore. M.J.Z. would like to thank Dr. R. Tavakoli for valuable discussion. Q. Z. would like to thank and Hongbo Lou, Fei Zhang and Yang Ren for help with synchrotron x-ray diffraction experiments and acknowledge the financial support from the DOE-BES X-ray Scattering Core Program under grant 
number DE-FG02-99ER45775 and from the National Natural Science Foundation of China under grant number U1530402.

\section{References}

[1] M.F. Ashby, and A.L. Greer, Metallic glasses as structural materials, Scripta Materialia. 54 (2006) 321-326.

[2] C.A. Schuh, T.C. Hufnagel, and U. Ramamurty, Mechanical behavior of amorphous alloys, Acta Materialia. 55 (2007) 4067-4109.

[3] F.F. Wu, W. Zheng, J.W. Deng, D.D. Qu, and J. Shen, Super-high compressive plastic deformation behaviors of Zr-based metallic glass at room temperature, Materials Science and Engineering: A. 541 (2012) 199-203.

[4] J. Das, M.B. Tang, K.B. Kim, R. Theissmann, F. Baier, W.H. Wang, and J. Eckert, Work-hardenable ductile bulk metallic glass, Physical Review Letters. 94 (2005) 205501.

[5] J. Schroers, and W.L. Johnson, Ductile bulk metallic glass, Physical Review Letters. 93 (2004) 255506.

[6] K.F. Yao, F. Ruan, Y.Q. Yang, and N. Chen, Superductile bulk metallic glass, Applied Physics Letters. 88 (2006) 122106.

[7] Y.H. Liu, G. Wang, R.J. Wang, d.e. .Q. Zhao, M.X. Pan, and W.H. Wang, Super plastic bulk metallic glasses at room temperature, Science. 315 (2007) 1385-8.

[8] H. Guo, P.F. Yan, Y.B. Wang, J. Tan, Z.F. Zhang, M.L. Sui, and E. Ma, Tensile ductility and necking of metallic glass, Nat Mater. 6 (2007) 735-9.

[9] D. Jang, and J.R. Greer, Transition from a strong-yet-brittle to a stronger-and-ductile state by size reduction of metallic glasses, Nature Materials. 9 (2010) 215-219.

[10] J.H. Luo, F.F. Wu, J.Y. Huang, J.Q. Wang, and S.X. Mao, Superelongation and atomic chain formation in nanosized metallic glass, Phys Rev Lett. 104 (2010) 215503.

[11] D.J. Magagnosc, R. Ehrbar, G. Kumar, M.R. He, J. Schroers, and D.S. Gianola, Tunable tensile ductility in metallic glasses, Sci Rep. 3 (2013) 1096.

[12] D.J. Magagnosc, G. Kumar, J. Schroers, P. Felfer, J.M. Cairney, and D.S. Gianola, Effect of ion irradiation on tensile ductility, strength and fictive temperature in metallic glass nanowires, Acta Materialia. 74 (2014) 165-182.

[13] D.Z. Chen, D. Jang, K.M. Guan, Q. An, W.A. Goddard, and J.R. Greer, Nanometallic glasses: size reduction brings ductility, surface state drives its extent, Nano Lett. 13 (2013) 4462-8.

[14] Y. Liu, H. Wu, C.T. Liu, Z. Zhang, and V. Keppens, Physical factors controlling the ductility of bulk metallic glasses, Applied Physics Letters. 93 (2008) 151915.

[15] J.J. Lewandowski*, W.H. Wang, and A.L. Greer, Intrinsic plasticity or brittleness of metallic glasses, Philosophical Magazine Letters. 85 (2005) 77-87.

[16] D. Toma, M. Meuris, and U. Köster, Oxidation of Zr-based metallic glasses in air, Journal of Non-crystalline Solids. 250 (1999) 719-723.

[17] Q. Jing, B. Zhang, J. Zhang, M. Ma, and R. Liu, Electrochemical corrosion and oxidation resistances of $\mathrm{Zr}_{60} \mathrm{Ni}_{21} \mathrm{Al}_{19}$ bulk amorphous alloys, Science China Physics, Mechanics and Astronomy. 53 (2010) 2223-2226.

[18] Y.H. Li, W. Zhang, C. Dong, J.B. Qiang, A. Makino, and A. Inoue, Formation and mechanical properties of Zr--Ni-Al glassy alloys with high glass-forming ability, Intermetallics. 18 (2010) 1851-1855.

[19] S. Sato, T. Sanada, J. Saida, M. Imafuku, E. Matsubara, and A. Inoue, Effect of Al on local structures of Zr-Ni and Zr-Cu metallic glasses, Materials Transactions. 46 (2005) 2893-2897.

[20] Q. Jing, Y. Zhang, D. Wang, and Y. Li, A study of the glass forming ability in ZrNiAl alloys, Materials Science and Engineering: A. 441 (2006) 106-111.

[21] K. Zhang, Y. Liu, J. Schroers, M.D. Shattuck, and C.S. O'Hern, The glass-forming ability of model metal-metalloid alloys, J Chem Phys. 142 (2015) 104504.

[22] S. Plimpton, Fast parallel algorithms for short-range molecular dynamics, Journal of Computational Physics. 117 (1995) 1-19. 
[23] Howard Sheng, School of Physics, Astronomy \& Computational Sciences, George Mason University, Fairfax, VA 22030 (Potential provided through private communications), (n.d.).

[24] D.J. Evans, and B.L. Holian, The nose--hoover thermostat, The Journal of Chemical Physics. 83 (1985) 40694074.

[25] H.C. Andersen, RATTLE: A Velocity version of the SHAKE algorithm for molecular dynamics calculations, Journal of Computational Physics. 52 (1983) 24-34.

[26] P.S. Branicio, and D.J. Srolovitz, Local stress calculation in simulations of multicomponent systems, Journal of Computational Physics. 228 (2009) 8467-8479.

[27] D.H. Tsai, The virial theorem and stress calculation in molecular dynamics, The Journal of Chemical Physics. 70 (1979) 1375-1382.

[28] F. Shimizu, S. Ogata, and J. Li, Theory of shear banding in metallic glasses and molecular dynamics calculations, Materials Transactions. 48 (2007) 2923-2927.

[29] A. Stukowski, Visualization and analysis of atomistic simulation data with OVITO--the Open Visualization Tool, Modelling and Simulation in Materials Science and Engineering. 18 (2010) 015012.

[30] J.L. Finney, Random packings and the structure of simple liquids. I. The geometry of random close packing, in: : Proceedings of the Royal Society of London A: Mathematical, Physical and Engineering Sciences, 1970: : p. $479-493$. [31] H.W. Sheng, W.K. Luo, F.M. Alamgir, J.M. Bai, and E. Ma, Atomic packing and short-to-medium-range order in metallic glasses, Nature. 439 (2006) 419-25.

[32] J. Ding, Y.-Q. Cheng, and E. Ma, Full icosahedra dominate local order in $\mathrm{Cu}_{64} \mathrm{Zr}_{34}$ metallic glass and supercooled liquid, Acta Materialia. 69 (2014) 343-354.

[33] A. Hirata, L.J. Kang, T. Fujita, B. Klumov, K. Matsue, M. Kotani, A.R. Yavari, and M.W. Chen, Geometric frustration of icosahedron in metallic glasses, Science. 341 (2013) 376-379.

[34] J. Lu, G. Ravichandran, and W.L. Johnson, Deformation behavior of the $\mathrm{Zr}_{41.2} \mathrm{Ti}_{13.8} \mathrm{Cu}_{12.5} \mathrm{Ni}_{10} \mathrm{Be}_{22.5}$ bulk metallic glass over a wide range of strain-rates and temperatures, Acta Materialia. 51 (2003) 3429-3443.

[35] Y.Q. Cheng, A.J. Cao, and E. Ma, Correlation between the elastic modulus and the intrinsic plastic behavior of metallic glasses: The roles of atomic configuration and alloy composition, Acta Materialia. 57 (2009) 3253-3267.

[36] A.J. Cao, Y.Q. Cheng, and E. Ma, Structural processes that initiate shear localization in metallic glass, Acta Materialia. 57 (2009) 5146-5155.

[37] P. Murali, T.F. Guo, Y.W. Zhang, R. Narasimhan, Y. Li, and H.J. Gao, Atomic scale fluctuations govern brittle fracture and cavitation behavior in metallic glasses, Phys Rev Lett. 107 (2011) 215501.

[38] X.W. Gu, M. Jafary-Zadeh, D.Z. Chen, Z. Wu, Y.W. Zhang, D.J. Srolovitz, and J.R. Greer, Mechanisms of failure in nanoscale metallic glass, Nano Lett. 14 (2014) 5858-64.

[39] S. Adibi, P.S. Branicio, R. Liontas, D.Z. Chen, J.R. Greer, D.J. Srolovitz, and S.P. Joshi, Surface roughness imparts tensile ductility to nanoscale metallic glasses, Extreme Mechanics Letters. (2015).

[40] S. Adibi, Z.-D. Sha, P.S. Branicio, S.P. Joshi, Z.-S. Liu, and Y.-W. Zhang, A transition from localized shear banding to homogeneous superplastic flow in nanoglass, Applied Physics Letters. 103 (2013) 211905.

[41] Z.D. Sha, L.C. He, Q.X. Pei, H. Pan, Z.S. Liu, Y.W. Zhang, and T.J. Wang, On the notch sensitivity of CuZr nanoglass, Journal of Applied Physics. 115 (2014) 163507.

[42] S.W. Lee, M. Jafary-Zadeh, D.Z. Chen, Y.W. Zhang, and J.R. Greer, Size Effect Suppresses Brittle Failure in Hollow $\mathrm{Cu}_{60} \mathrm{Zr}_{40}$ Metallic Glass Nanolattices Deformed at Cryogenic Temperatures, Nano Lett. 15 (2015) 5673-81.

[43] Y.Q. Cheng, A.J. Cao, H.W. Sheng, and E. Ma, Local order influences initiation of plastic flow in metallic glass: Effects of alloy composition and sample cooling history, Acta Materialia. 56 (2008) 5263-5275.

[44] J. Ding, S. Patinet, M.L. Falk, Y. Cheng, and E. Ma, Soft spots and their structural signature in a metallic glass, Proceedings of the National Academy of Sciences. 111 (2014) 14052-14056.

[45] L. Zhang, Y.-Q. Cheng, A. Cao, J. Xu, and E. Ma, Bulk metallic glasses with large plasticity: Composition design from the structural perspective, Acta Materialia. 57 (2009) 1154-1164.

[46] X.W. Gu, C.N. Loynachan, Z. Wu, Y.W. Zhang, D.J. Srolovitz, and J.R. Greer, Size-dependent deformation of nanocrystalline Pt nanopillars, Nano Lett. 12 (2012) 6385-92.

[47] H. Zhang, B.E. Schuster, Q. Wei, and K.T. Ramesh, The design of accurate micro-compression experiments, Scripta Materialia. 54 (2006) 181-186.

[48] D. Kiener, and A.M. Minor, Source truncation and exhaustion: insights from quantitative in situ TEM tensile testing, Nano Lett. 11 (2011) 3816-20. 
[49] A.R. Yavari, A. Le Moulec, A. Inoue, N. Nishiyama, N. Lupu, E. Matsubara, W.J. Botta, G. Vaughan, M. Di Michiel, and Kvick, Excess free volume in metallic glasses measured by X-ray diffraction, Acta Materialia. 53 (2005) 1611-1619.

[50] A.R. Yavari, M. Tonegaru, N. Lupu, A. Inoue, E. Matsubara, G. Vaughan, Kvick, and W.J. Botta, Quenched-in Free Volume V f, Deformation-induced Free Volume, the Glass Transition Tg and Thermal Expansion in glassy ZrNbCuNiAl measured by Time-resolved Diffraction in Transmission, in: : MRS Proceedings, 2003: : p. MM3-5.

[51] E. Teatum, K. Gschneidner Jr, and J. Waber, Compilation of calculated data useful in predicting metallurgical behavior of the elements in binary alloy systems, 1959.

[52] U. Ramamurty, M.L. Lee, J. Basu, and Y. Li, Embrittlement of a bulk metallic glass due to low-temperature annealing, Scripta Materialia. 47 (2002) 107-111.

[53] F.H. Stillinger, A topographic view of supercooled liquids and glass formation, Science. 267 (1995) $1935-9$.

[54] P. Murali, and U. Ramamurty, Embrittlement of a bulk metallic glass due to sub-T g annealing, Acta Materialia. 53 (2005) 1467-1478.

[55] D. Suh, and R.H. Dauskardt, Mechanical relaxation time scales in a Zr--Ti--Ni--Cu--Be bulk metallic glass, Journal of Materials Research. 17 (2002) 1254-1257.

[56] Y. Fan, T. Iwashita, and T. Egami, How thermally activated deformation starts in metallic glass, Nat Commun. 5 (2014) 5083.

[57] C.A. Volkert, A. Donohue, and F. Spaepen, Effect of sample size on deformation in amorphous metals, Journal of Applied Physics. 103 (2008) 083539-083539.

[58] A. Van den Beukel, and J. Sietsma, The glass transition as a free volume related kinetic phenomenon, Acta Metallurgica Et Materialia. 38 (1990) 383-389.

[59] Q.-K. Li, and M. Li, Assessing the critical sizes for shear band formation in metallic glasses from molecular dynamics simulation, Applied Physics Letters. 91 (2007) 231905.

[60] F. Delogu, Molecular dynamics study of size effects in the compression of metallic glass nanowires, Physical Review B. 79 (2009) 184109.

[61] T. Mukai, T.G. Nieh, Y. Kawamura, A. Inoue, and K. Higashi, Effect of strain rate on compressive behavior of a $\mathrm{Pd}_{40} \mathrm{Ni}_{40} \mathrm{P}_{20}$ bulk metallic glass, Intermetallics. 10 (2002) 1071-1077.

[62] L. Tian, Z.-W. Shan, and E. Ma, Ductile necking behavior of nanoscale metallic glasses under uniaxial tension at room temperature, Acta Materialia. 61 (2013) 4823-4830.

[63] C.A. Volkert, and A.M. Minor, Focused ion beam microscopy and micromachining, MRS Bulletin. 32 (2007) 389-399.

[64] G. Moser, H. Felber, B. Rashkova, P.J. Imrich, C. Kirchlechner, W. Grosinger, C. Motz, G. Dehm, and D. Kiener, Sample preparation by metallography and focused ion beam for nanomechanical testing, Practical Metallography. 49 (2012) 343-355.

[65] D. Kiener, C. Motz, M. Rester, M. Jenko, and G. Dehm, FIB damage of Cu and possible consequences for miniaturized mechanical tests, Materials Science and Engineering: A. 459 (2007) 262-272.

[66] S. Nagata, S. Higashi, B. Tsuchiya, K. Toh, T. Shikama, K. Takahiro, K. Ozaki, K. Kawatusra, S. Yamamoto, and A. Inouye, Ion irradiation effects on amorphization and thermal crystallization in Zr--Al--Ni--Cu alloys, Nuclear Instruments and Methods in Physics Research Section B: Beam Interactions with Materials and Atoms. 257 (2007) 420-423.

[67] T. Nagase, and Y. Umakoshi, Electron irradiation induced nanocrystallization behavior in Fe71Zr9B20 metallic glass, Materials Transactions. 46 (2005) 608-615.

[68] J. Tao, Z. Yang, X. Mu, C. Chen, and W. Xie, Influences of ion implantation on non-isothermal crystallization behaviors of bulk metallic glass, Journal of Alloys and Compounds. 479 (2009) 736-740.

[69] H. Gao, B. Ji, I.L. Jager, E. Arzt, and P. Fratzl, Materials become insensitive to flaws at nanoscale: lessons from nature, Proc Natl Acad Sci U S A. 100 (2003) 5597-600.

[70] R. Liontas, X.W. Gu, E. Fu, Y. Wang, N. Li, N. Mara, and J.R. Greer, Effects of helium implantation on the tensile properties and microstructure of $\mathrm{Ni}_{73} \mathrm{P}_{27}$ metallic glass nanostructures, Nano Lett. 14 (2014) 5176-83. 
Figure 1. Fabrication process for nano-tensile specimens. (1) A commercially available Si TEM grid is used as the substrate, (2) Zr-Ni-Al metallic glass is sputtered onto the TEM grid, with annealing conducted under an Ar environment in a tube furnace, (3) insulator enhanced etching of Si with $\mathrm{XeF}_{2}$ gas in an SEM used to selectively remove Si from the top section of the posts on the TEM grid, (4) the resultant free-standing film of $\mathrm{Zr}-\mathrm{Ni}$-Al on the posts of the TEM grid, FIB-milled into individual nano-tensile specimens by extensively tilting/rotating the SEM stage to allow the appropriate FIB-removal of material. SEM images of the nanomechanical testing specimens are shown from both top-down and side view at $\sim 5^{\circ}$ tilt

Figure 2. TEM and EDS elemental analysis on (a-c) as-sputtered and ( $\mathbf{d}-\mathbf{g}$ ) annealed $\mathrm{Zr}-\mathrm{Ni}-\mathrm{Al}$ metallic glass, including (a) diffraction pattern from as-sputtered film, (b) high-resolution TEM image of the as-sputtered film, (c) EDS elemental analysis along the depth of the $\sim 4.5 \mu \mathrm{m}$ thick as-sputtered film with error denoting $\pm 1 \sigma$ in the measurement, (d) diffraction pattern from test section of the annealed nano-mechanical specimen, (e) TEM image of the annealed nanotensile specimen, (f) dark-field image on the surface edge of the annealed nano-tensile specimen, and (g) EDS elemental analysis on annealed nano-tensile specimens with error denoting $\pm 1 \sigma$ in the measurement.

Figure 3. Uniaxial tensile experiments on the as-sputtered $\mathrm{Zr}$-Ni-Al metallic glass samples, including (left) engineering stress-strain data, (center) true stress-strain data, and (a-d) SEM images from in-situ videos around the time of sample failure. The data is grouped by specimen size with $\sim 90 \mathrm{~nm}$ wide specimens in the top row, $\sim 110 \mathrm{~nm}$ wide specimens in the top-middle row, $\sim 150 \mathrm{~nm}$ wide specimens in the bottom-middle row, and $\sim 200 \mathrm{~nm}$ wide specimens in the bottom row.

Figure 4. Uniaxial tensile results on annealed Zr-Ni-Al metallic glass including (left) engineering stress-strain data, (center) true stress-strain data, and (a-d) SEM images from in-situ videos around the time of sample failure. The data is grouped by specimen size with $\sim 90 \mathrm{~nm}$ wide specimens in the top row, $110 \mathrm{~nm}$ wide specimens in the top-middle row, $150 \mathrm{~nm}$ wide specimens in the bottom-middle row, and $\sim 200 \mathrm{~nm}$ wide specimens in the bottom row.

Figure 5. Mechanical properties of as-sputtered (denoted by blue squares) and annealed (denoted by red diamonds) nano-tensile specimens: (a) engineering plastic strain, (b) true plastic strain, (c) yield strength, (d) ultimate tensile strength, (e) engineering yield strain, (f) elastic modulus. The vertical error bars denote $\pm 1 \sigma$ in the measured property and the horizontal error bars denote $\pm 1 \sigma$ in the pillar widths measured for each size grouping ( $90 \mathrm{~nm}$, $\sim 110 \mathrm{~nm}, \sim 150 \mathrm{~nm}, \sim 200 \mathrm{~nm})$.

Figure 6. Synchrotron XRD results on as-sputtered and annealed at $625 \mathrm{~K} \mathrm{Zr-Ni-Al}$ metallic glass including: (a) structure factor, (b) zoomed-in region on the principal peak of the structure factor shown in (a) with dashed lines denoting the peak positions, (c) zoomed-in region on the second 
peak of the structure factor shown in (a), (d) reduced pair distribution function, (e) zoomed-in region on the reduced pair distribution function shown in (d) at $r \leq 2.25 \AA$ with dashed lines denoting the slope of the curve in this low-r region, (f) zoomed-in region on the first nearestneighbor peak of the reduced pair distribution function shown in (d) with dashed lines denoting metallic bond distances shown in Table 1.

Figure 7. Molecular dynamics simulation on the effect of cooling-rate on the mechanical behaviour and atomic structure of Zr-Ni-Al metallic glass specimens with diameters of $40 \mathrm{~nm}$ and heights of $120 \mathrm{~nm}$. (a) Tensile engineering stress versus strain obtained at room temperature for samples formed with cooling rates denoted in the legend. (b-d) Analysis of the atomics von Mises shear strains $\left(\varepsilon^{\mathrm{vM}}\right)$ of the samples formed with the cooling rates of (b) $10^{10}$ $\mathrm{K} / \mathrm{s}$, (c) $10^{11} \mathrm{~K} / \mathrm{s}$, (d) $10^{12} \mathrm{~K} / \mathrm{s}$, and (e) $10^{13} \mathrm{~K} / \mathrm{s}$. (f) Effect of cooling rate on the average atomic potential energy (PE) of the system (left axis) as well as the fraction of Al-centered fullicosahedra clusters (right axis).

Figure 8. Molecular dynamics simulations on the effect of annealing at $T=850 \mathrm{~K}(80 \%$ of simulated $\mathrm{T}_{\mathrm{g}}$ ) on the mechanical behavior and atomic structure of $\mathrm{Zr}-\mathrm{Ni}$-Al metallic glass specimens with diameters of $40 \mathrm{~nm}$ and heights of $120 \mathrm{~nm}$. (a) Tensile engineering stress-strain response of the initial as-quenched sample formed with a cooling rate of $10^{13} \mathrm{~K} / \mathrm{s}$, after annealing at $200 \mathrm{~ns}$, after annealing at $400 \mathrm{~ns}$, and for comparison the sample formed with a cooling rate of $10^{10} \mathrm{~K} / \mathrm{s}$. (b-c) Visualization of atomic von Mises shear strains of the samples annealed for (b) $200 \mathrm{~ns}$ and (c) $400 \mathrm{~ns}$. (d) Structural evolution of the metallic glass during annealing at $850 \mathrm{~K}$ as measured by the average atomic potential energy (PE) of the system (left axis) as well as the fraction of Al-centered full-icosahedra clusters (right axis)

Figure 9. Molecular dynamics simulations on the effect of sample size (i.e. nanopillar diameter) on the mechanical behaviour of the $\mathrm{Zr}-\mathrm{Ni}$-Al metallic glass. The height of the nanopillars was selected such that the aspect ratio was constant at a value of 3 for height to diameter. (a) Tensile engineering stress versus strain for samples with diameters denoted in the legend (b-e) Analysis of the atomic von Mises shear strain $\left(\varepsilon^{\mathrm{vM}}\right)$ of the nanopillar samples with the diameters of (b) $40 \mathrm{~nm}$, (c) $20 \mathrm{~nm}$, (d) $10 \mathrm{~nm}$, and (e) $5 \mathrm{~nm}$ indicates a transition from brittle shear banding to ductile necking by decreasing the sample size.

Video 1A. Synched engineering stress-strain response with corresponding in-situ video obtained from the tensile testing of a 212-nm wide as-sputtered nano-tensile specimen. The video is played back at a speed 10x faster than it was captured.

Video 1B. Synched true stress-strain response with corresponding in-situ video obtained from the tensile testing of a 212-nm wide as-sputtered nano-tensile specimen. The video is played back at a speed 10x faster than it was captured.

Video 2A. Synched engineering stress-strain response with corresponding in-situ video obtained from the tensile testing of a 114-nm wide as-sputtered nano-tensile specimen. The video is played back at a speed 10x faster than it was captured. 
Video 2B. Synched true stress-strain response with corresponding in-situ video obtained from the tensile testing of a 114-nm wide as-sputtered nano-tensile specimen. The video is played back at a speed 10x faster than it was captured.

Video 3A. Synched engineering stress-strain response with corresponding in-situ video obtained from the tensile testing of a 209-nm wide annealed nano-tensile specimen. The video is played back at a speed $10 x$ faster than it was captured.

Video 3B. Synched true stress-strain response with corresponding in-situ video obtained from the tensile testing of a 209-nm wide annealed nano-tensile specimen. The video is played back at a speed $10 x$ faster than it was captured.

Video 4A. Synched engineering stress-strain response with corresponding in-situ video obtained from the tensile testing of a 109-nm wide annealed nano-tensile specimen. The video is played back at a speed $10 x$ faster than it was captured.

Video 4B. Synched true stress-strain response with corresponding in-situ video obtained from the tensile testing of a 109-nm wide annealed nano-tensile specimen. The video is played back at a speed $10 x$ faster than it was captured.

Video 5A. Synched engineering stress-strain response with corresponding in-situ video obtained from the tensile testing of a 93-nm wide annealed nano-tensile specimen. The video is played back at a speed 10x faster than it was captured.

Video 5B. Synched true stress-strain response with corresponding in-situ video obtained from the tensile testing of a 93-nm wide annealed nano-tensile specimen. The video is played back at a speed $10 x$ faster than it was captured. 
(1) Utilize Si TEM grid as a substrate

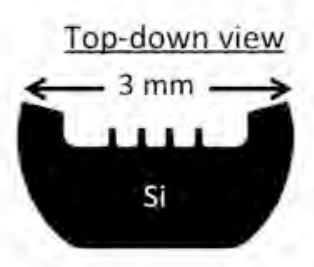

Side view

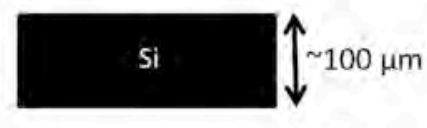

(2) Sputter $\mathrm{Zr}$-Ni-Al metallic glass (and conduct annealing)

Top-down view

Iu, I

ZaNizAl

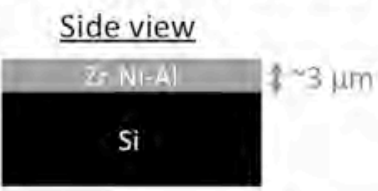

$\mathrm{Si}$

(3) Insulator enhanced etching of Si with $\mathrm{XeF}_{2}$ gas

Top-down view

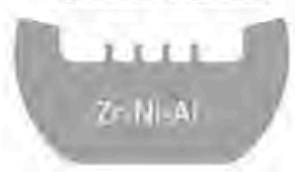

Side view

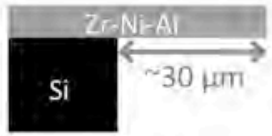

(4) FIB-milling of tensile nanomechanical testing specimens

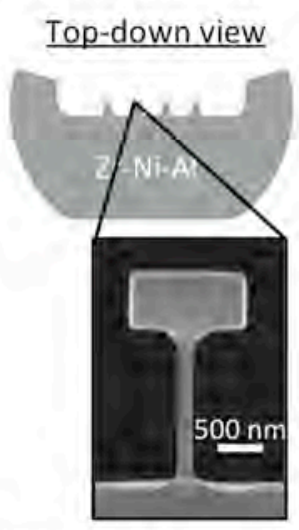

Side view
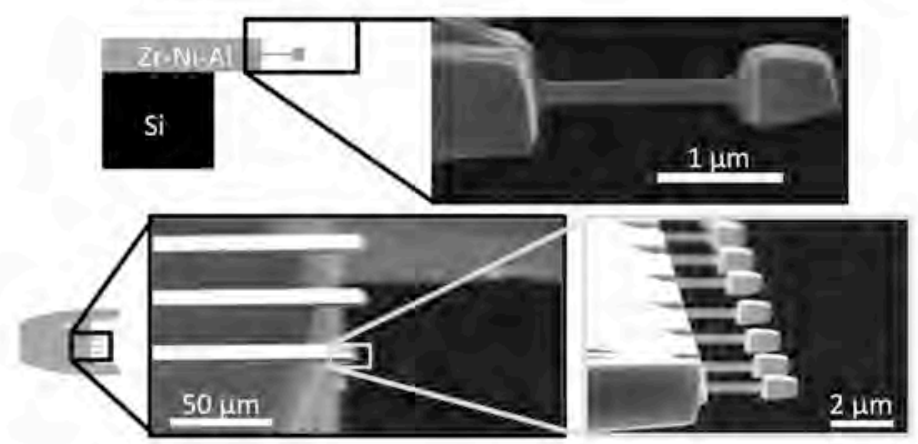

Figure 1. Fabrication process for nano-tensile nanomechanical specimens. (1) A commercially available Si TEM grid is used as the substrate, (2) $\mathrm{Zr}$ - $\mathrm{Ni}$-Al metallic glass is sputtered onto the TEM grid, with annealing conducted under an Ar environment in a tube furnace, (3) insulator enhanced etching of Si with $\mathrm{XeF}_{2}$ gas in an SEM used to selectively remove Si from the top section of the posts on the TEM grid, (4) the resultant free-standing film of $\mathrm{Zr}$ - $\mathrm{Ni}$-Al on the posts of the TEM grid, FIB-milled into individual nano-tensile specimens by extensively tilting/rotating the SEM stage to allow the appropriate FIB-removal of material. SEM images of the nanomechanical testing specimens are shown from both top-down and side view at $\sim 5^{\circ}$ tilt 

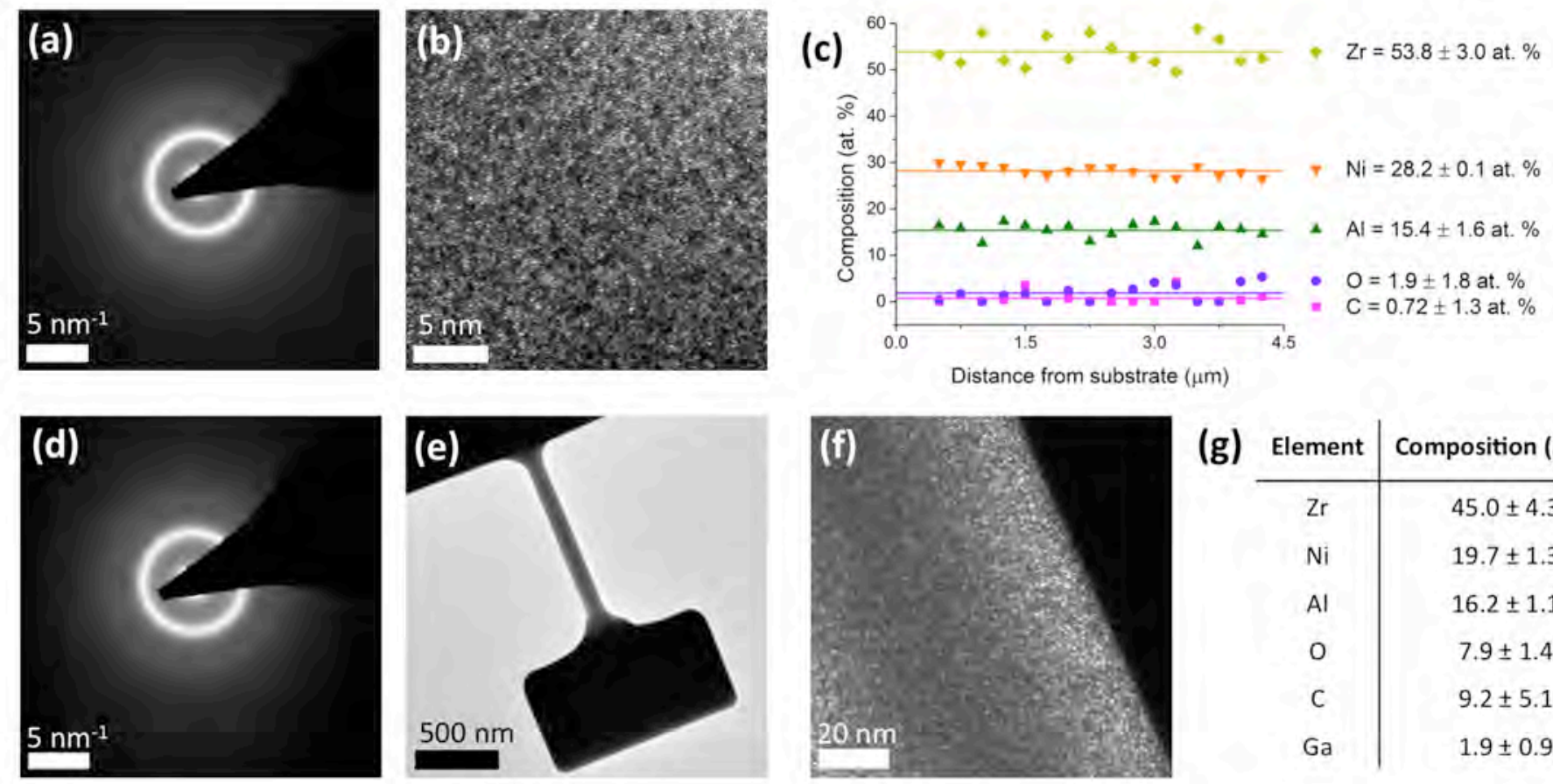

\begin{tabular}{c|c} 
(g) Element & Composition (at. \%) \\
\hline $\mathrm{Zr}$ & $45.0 \pm 4.3$ \\
$\mathrm{Ni}$ & $19.7 \pm 1.3$ \\
$\mathrm{Al}$ & $16.2 \pm 1.1$ \\
$\mathrm{O}$ & $7.9 \pm 1.4$ \\
$\mathrm{C}$ & $9.2 \pm 5.1$ \\
$\mathrm{Ga}$ & $1.9 \pm 0.9$
\end{tabular}

Figure 2. TEM and EDS elemental analysis on (a-c) as-sputtered and (d-g) annealed $\mathrm{Zr}-\mathrm{Ni}$-Al metallic glass, including (a) diffraction pattern from as-sputtered film, (b) high-resolution TEM image of the as-sputtered film, (c) EDS elemental analysis along the depth of the $\sim 4.5 \mu \mathrm{m}$ thick as-sputtered film, (d) diffraction pattern from test section of the annealed nano-mechanical specimen, (e) TEM image of the annealed nano-mechanical testing specimen, and (f) dark-field image on the surface edge of the annealed nano-mechanical testing specimen. 
- annealed

(a)

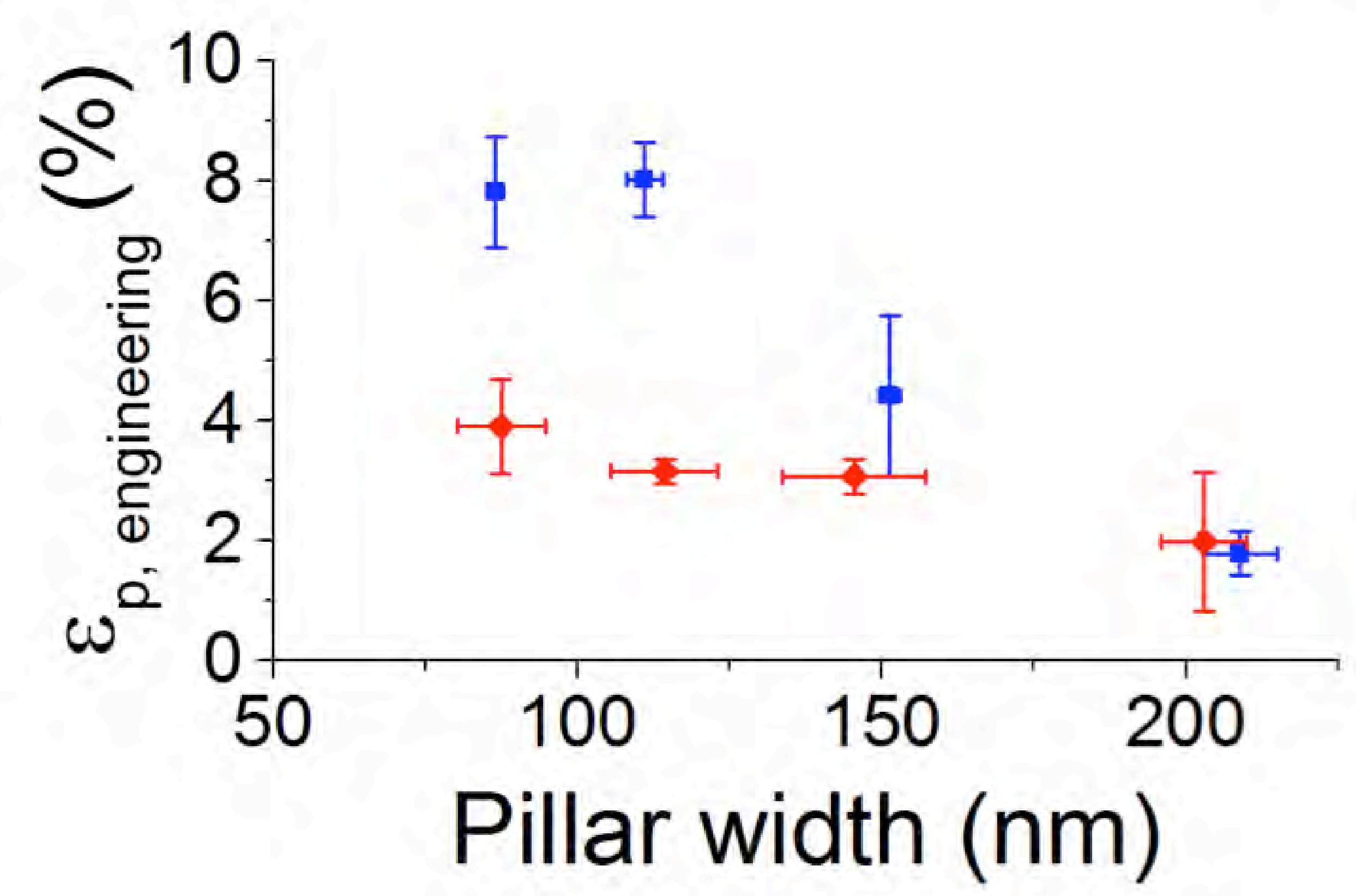

(c)

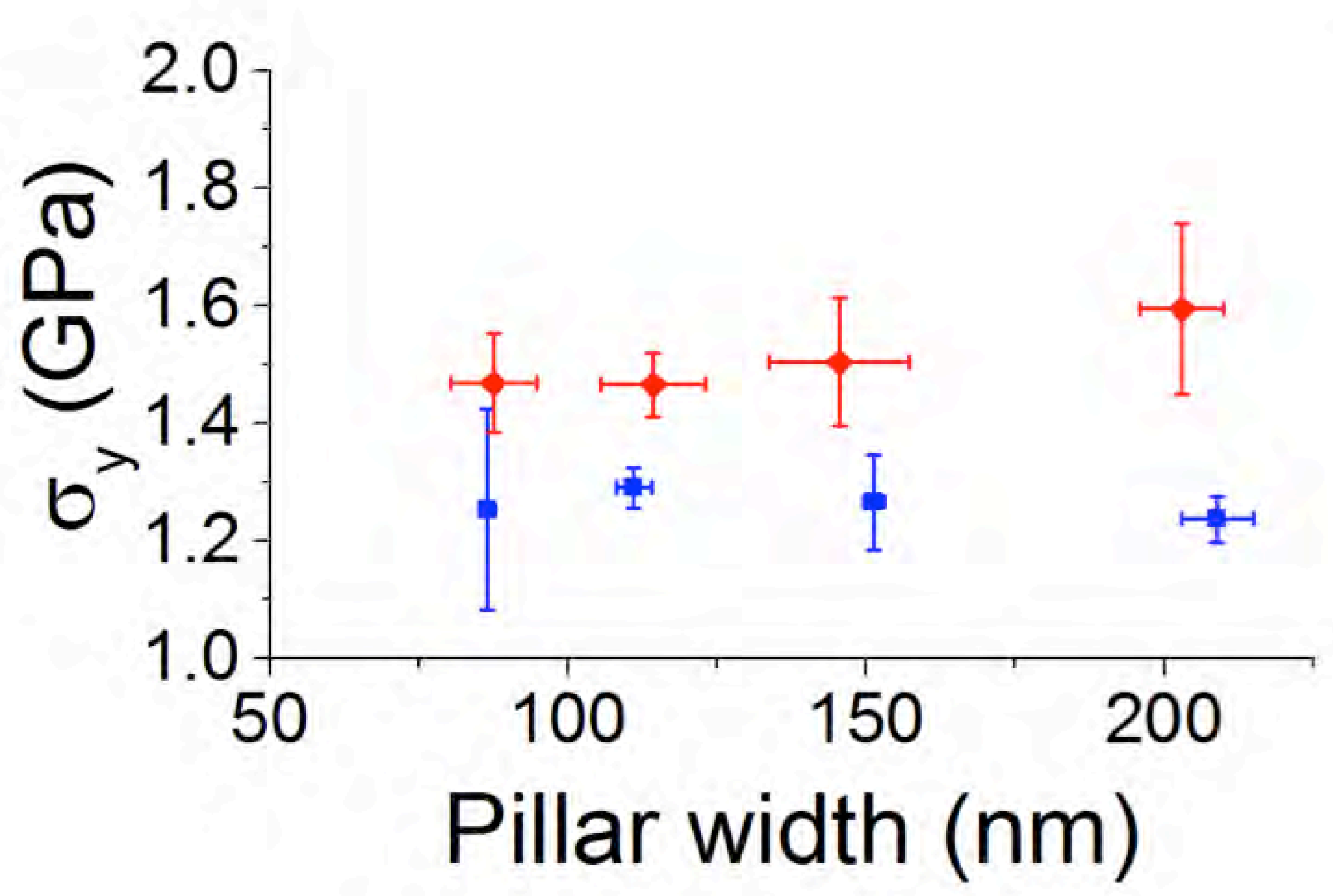

(e)

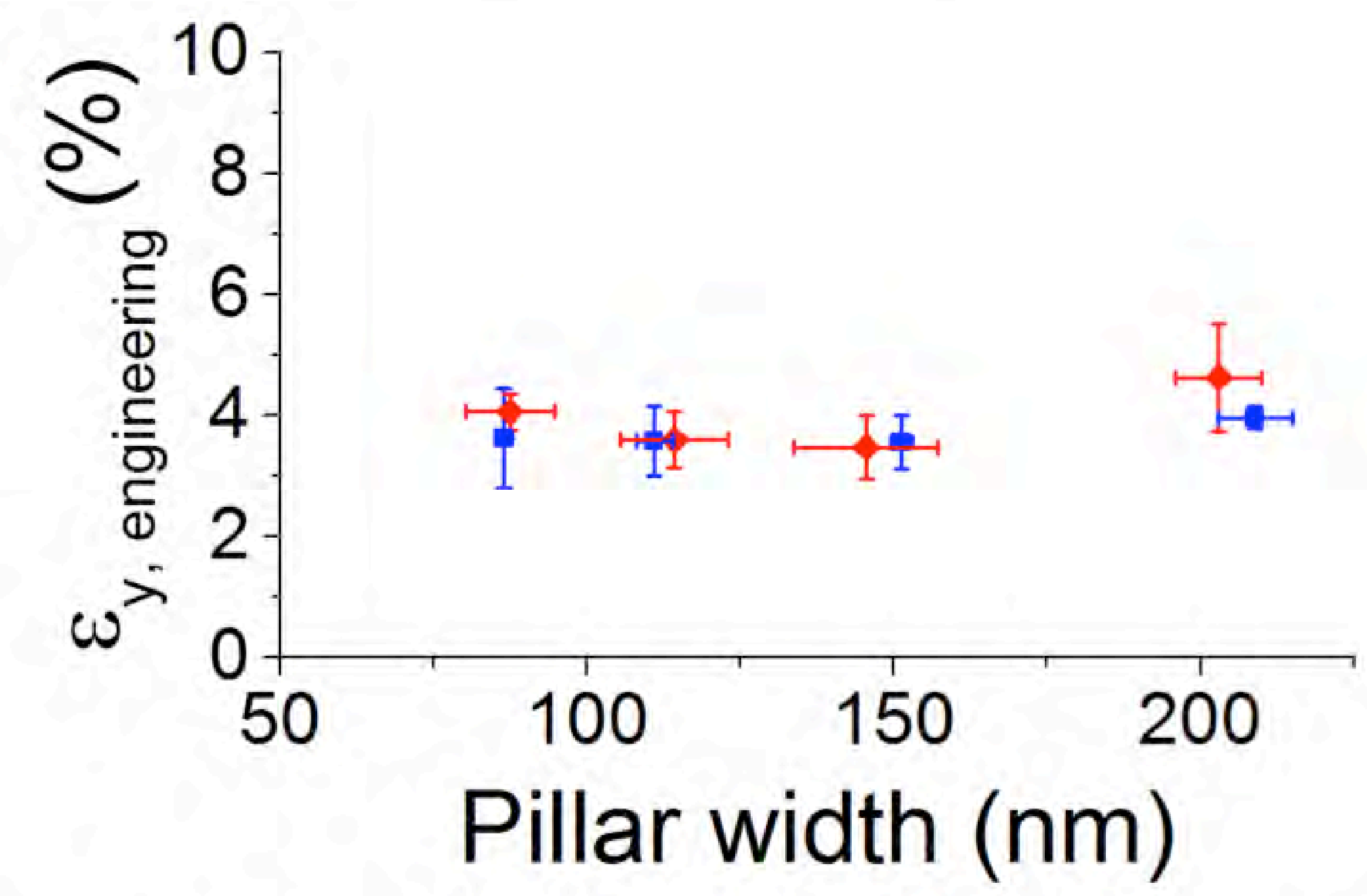

(b)

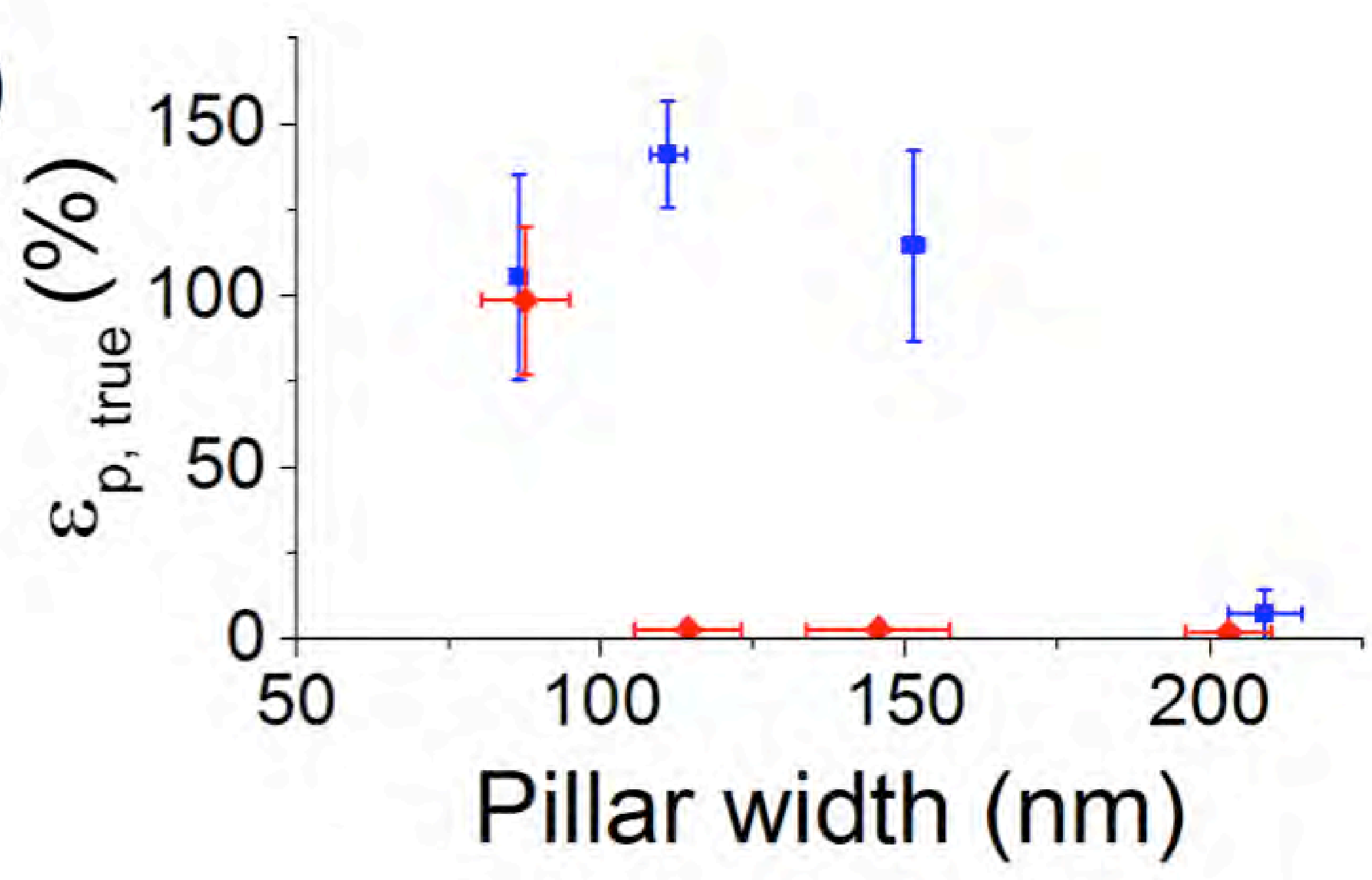

(d)

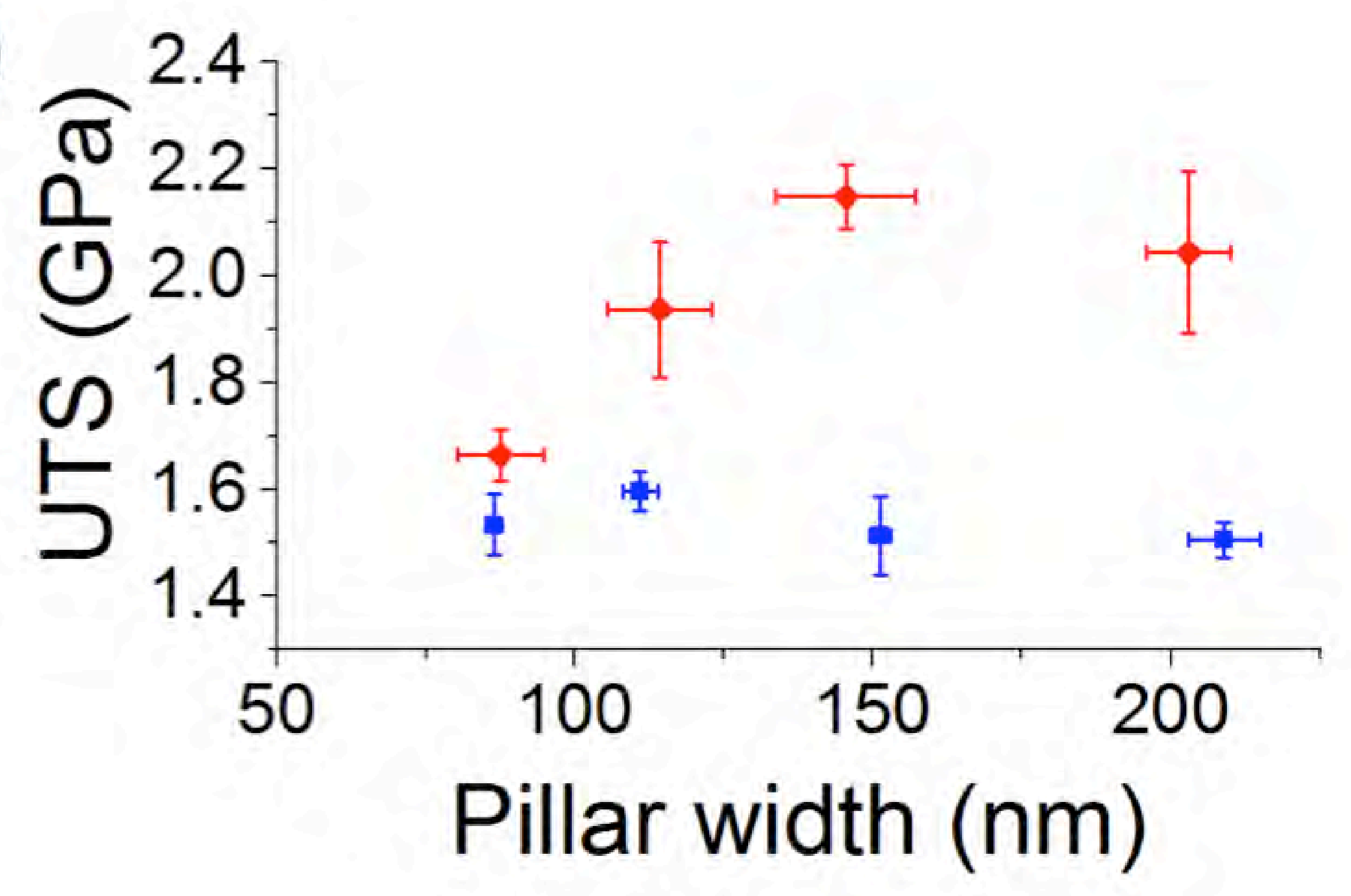

(f)

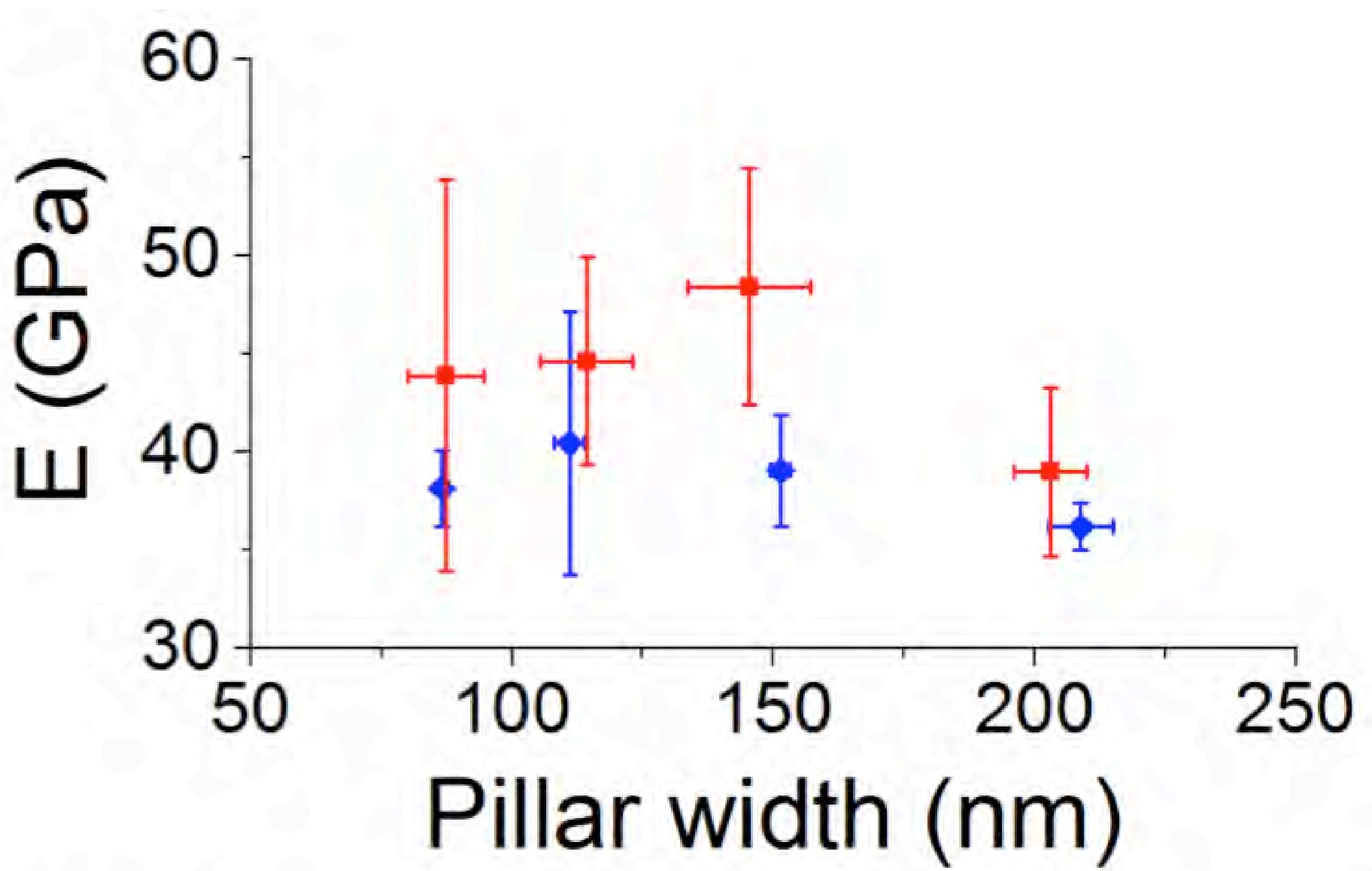

Figure 5. Mechanical properties of as-sputtered (denoted by blue squares) and annealed (denoted by red diamonds) nano-tensile specimens: (a) engineering plastic strain, (b) true plastic strain, (c) yield strength, (d) ultimate tensile strength, (e) engineering yield strain, (f) elastic modulus. The vertical error bars denote standard deviation in the measured property and the horizontal error bars denote standard deviation in the pillar width of each size grouping ( $\sim 90 \mathrm{~nm}, \sim 110 \mathrm{~nm}, \sim 150 \mathrm{~nm}, \sim 200 \mathrm{~nm}$ ) . 

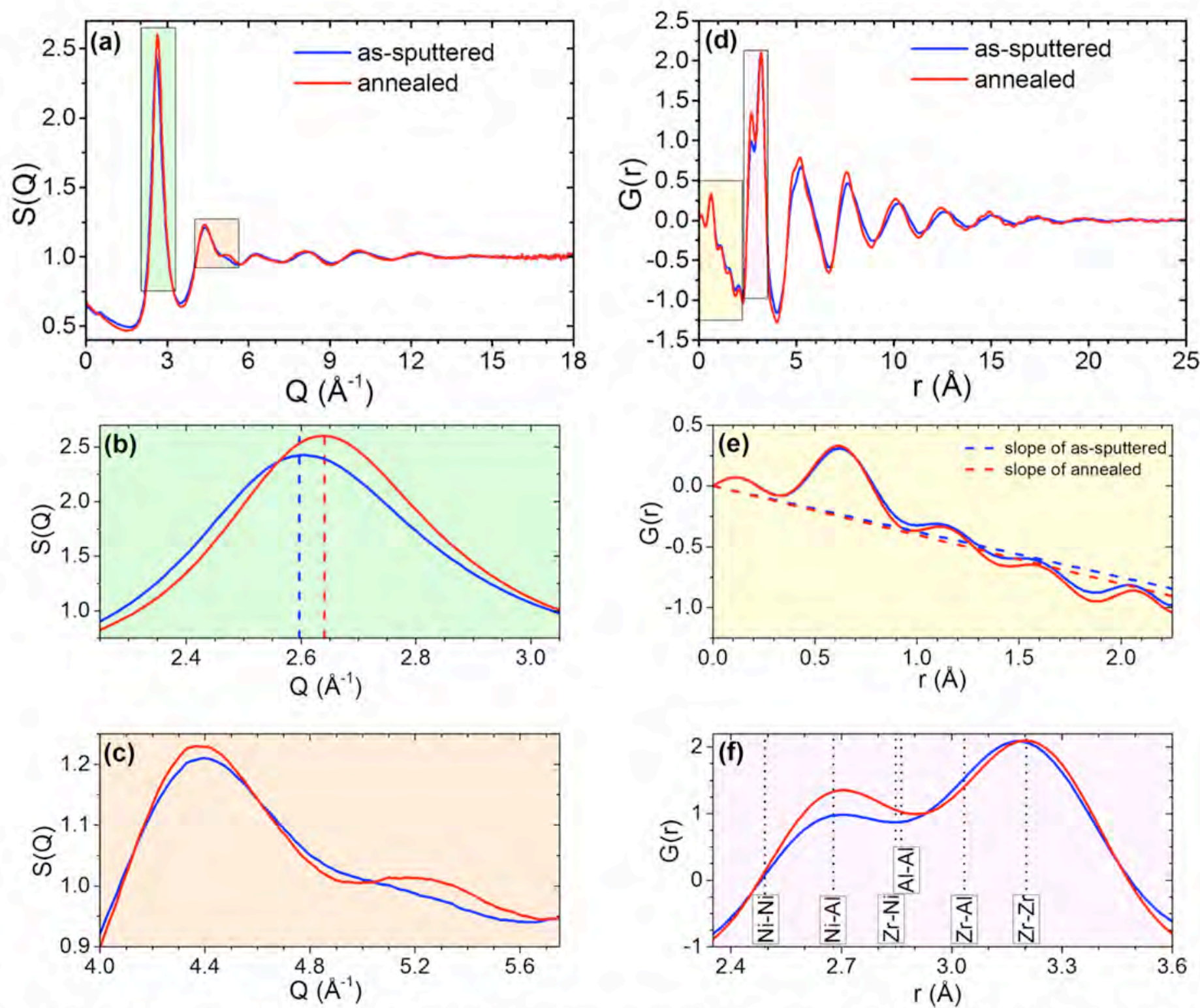

Figure 6. Synchrotron XRD results on as-sputtered and annealed at $625 \mathrm{~K} \mathrm{Zr-Ni-Al}$ metallic glass including: (a) structure factor, (b) zoomed-in region on the principal peak of the structure factor shown in (a) with dashed lines denoting the peak positions, (c) zoomed-in region on the second peak of the structure factor shown in (a), (d) reduced pair distribution function, (e) zoomed-in region on the reduced pair distribution function shown in (d) at $r \leq 2.25 \AA$ with dashed lines denoting the slope of the curve in this low-r region, (f) zoomed-in region on the first nearest-neighbor peak of the reduced pair distribution function shown in (d) with dashed lines denoting metallic bond distances shown in Table 1. 


\section{(a)}

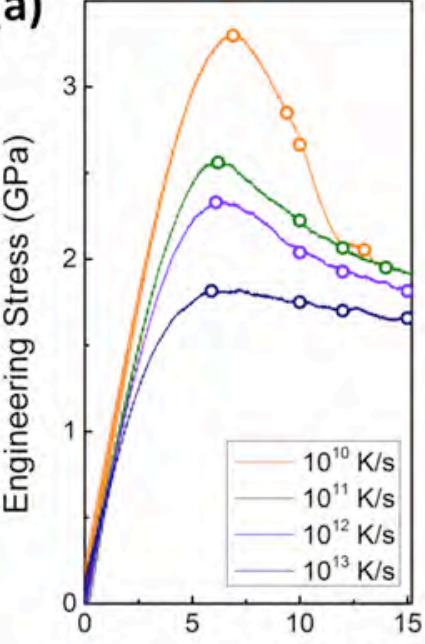

Engineering Strain (\%) (b) $10^{10} \mathrm{~K} / \mathrm{s}$

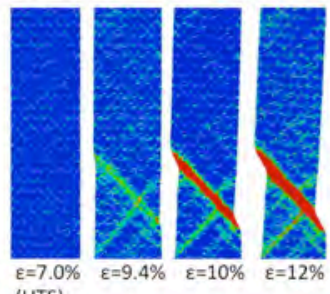

(UTS)
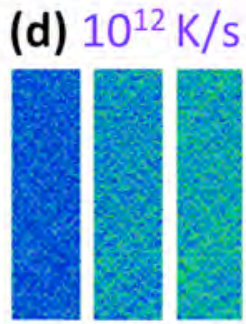

$\varepsilon=6.1 \% \quad \varepsilon=10 \% \quad \varepsilon=12 \% \quad \varepsilon=15 \%$ (UTS) (c) $10^{11} \mathrm{~K} / \mathrm{s}$

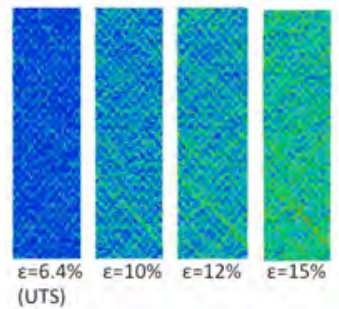

0.6

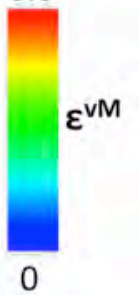

(f)

- Potential energy per atom

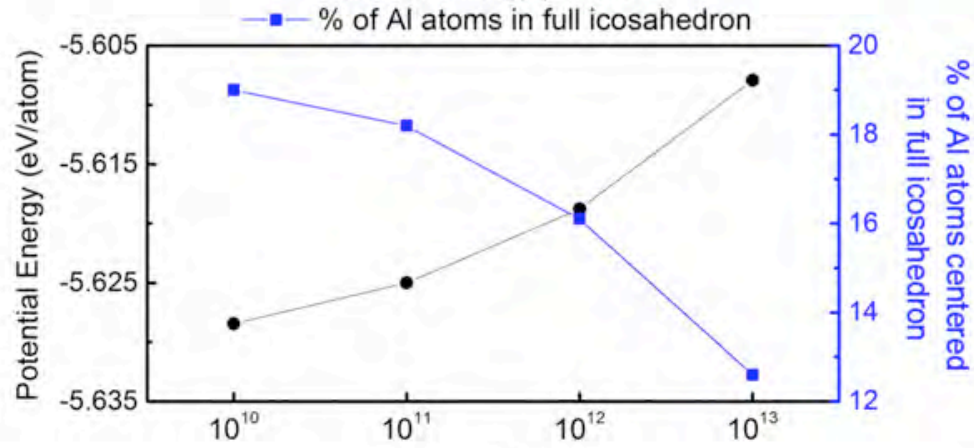

Cooling Rate $(\mathrm{K} / \mathrm{s})$

Figure 7. Molecular dynamics simulation on the effect of cooling-rate on the mechanical behaviour and atomic structure of $\mathrm{Zr}-\mathrm{Ni}$-Al metallic glass specimens with diameters of $40 \mathrm{~nm}$ and heights of $120 \mathrm{~nm}$. (a) Tensile engineering stress versus strain obtained at room temperature for samples formed with cooling rates denoted in the legend. (b-d) Analysis of the atomics von Mises shear strains $\left(\varepsilon^{\mathrm{vM}}\right)$ of the samples formed with the cooling rates of (b) $10^{10} \mathrm{~K} / \mathrm{s}$, (c) $10^{11} \mathrm{~K} / \mathrm{s}$, (d) $10^{12} \mathrm{~K} / \mathrm{s}$, and (e) $10^{13} \mathrm{~K} / \mathrm{s}$. (f) Effect of cooling rate on the average atomic potential energy (PE) of the system (left axis) as well as the fraction of Al-centered full-icosahedra clusters (right axis). 
(a)

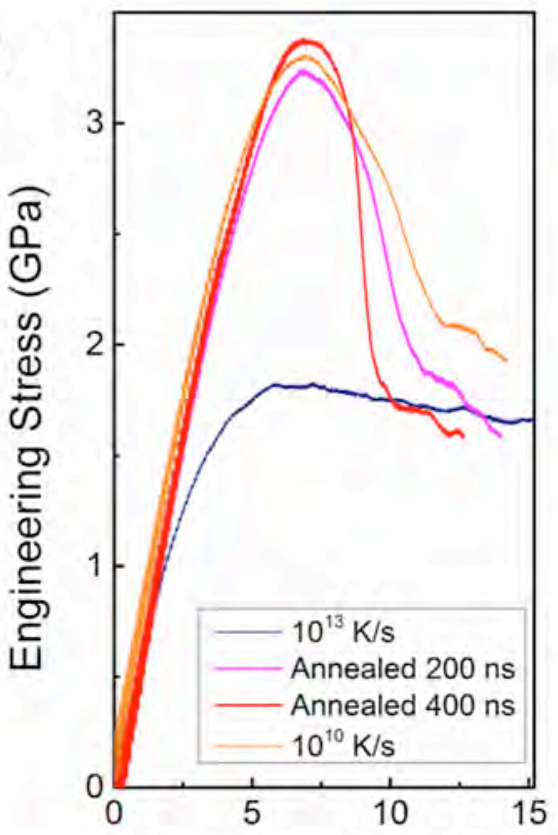

Engineering Strain (\%) (b) $200 \mathrm{~ns}$

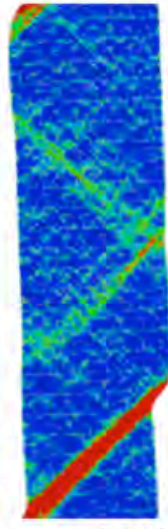

$\varepsilon=10 \%$

0

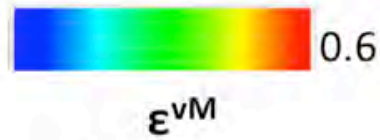

(c) $400 \mathrm{~ns}$

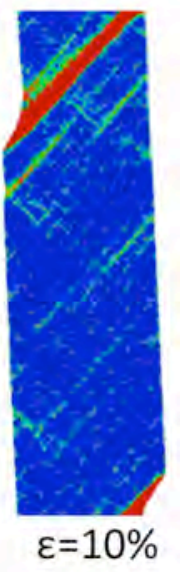

0.6

(d)

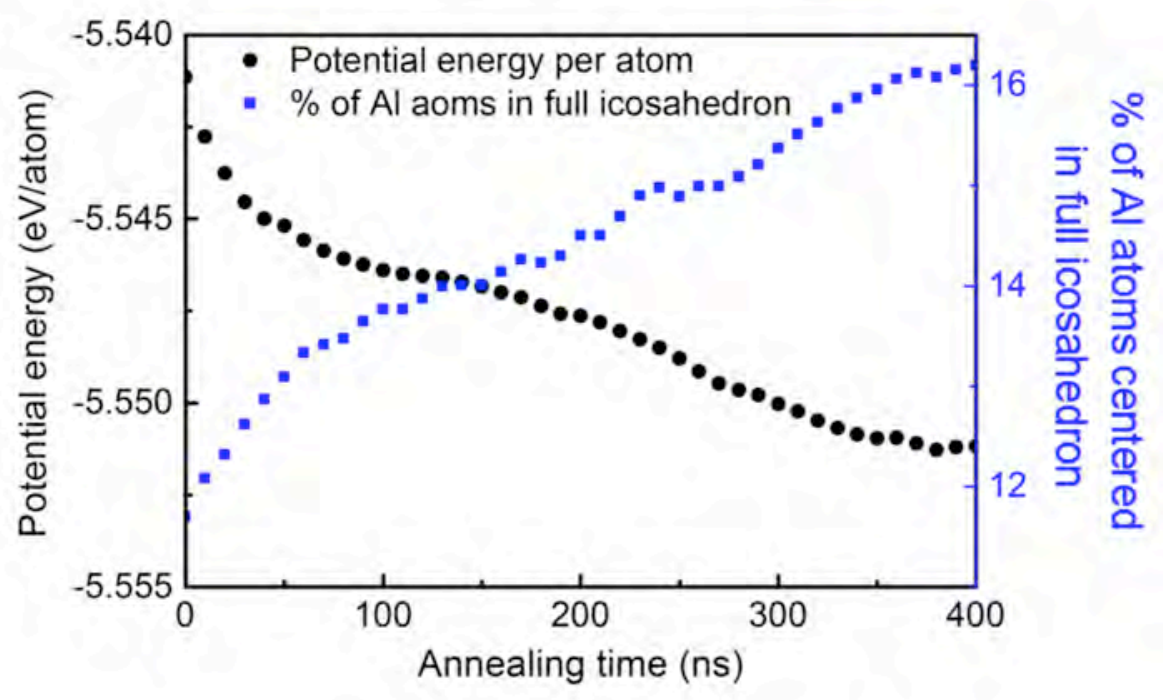

Figure 8. Molecular dynamics simulations on the effect of annealing at $\mathrm{T}=850 \mathrm{~K}\left(80 \%\right.$ of simulated $\mathrm{T}_{\mathrm{g}}$ ) on the mechanical behavior and atomic structure of $\mathrm{Zr}$ - Ni-Al metallic glass specimens with diameters of $40 \mathrm{~nm}$ and heights of $120 \mathrm{~nm}$. (a) Tensile engineering stress-strain response of the initial as-quenched sample formed with a cooling rate of $10^{13} \mathrm{~K} / \mathrm{s}$, after annealing at $200 \mathrm{~ns}$, after annealing at $400 \mathrm{~ns}$, and for comparison the sample formed with a cooling rate of $10^{10} \mathrm{~K} /$ s. (b-c) Visualization of atomic von Mises shear strains of the samples annealed for (b) $200 \mathrm{~ns}$ and (c) 400 ns. (d) Structural evolution of the metallic glass during annealing at $850 \mathrm{~K}$ as measured by the average atomic potential energy (PE) of the system (left axis) as well as the fraction of Al-centered full-icosahedra clusters (right axis) 
(a)

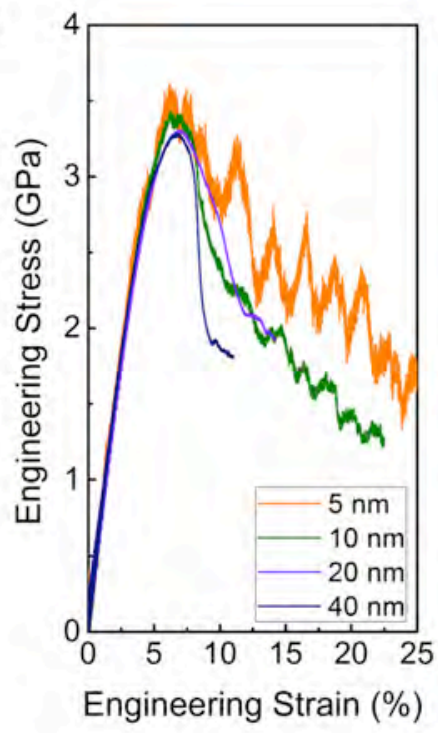

(b)

$40 \mathrm{~nm}$
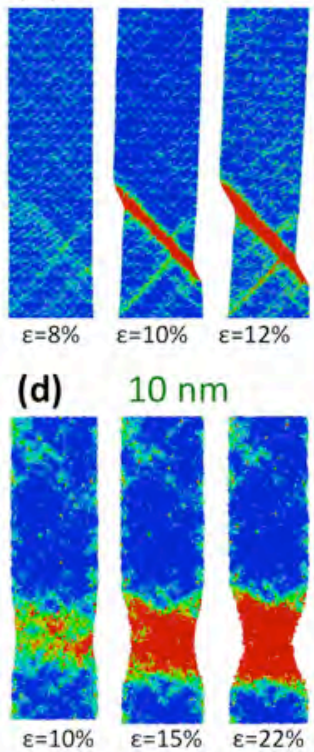

(c) $20 \mathrm{~nm}$

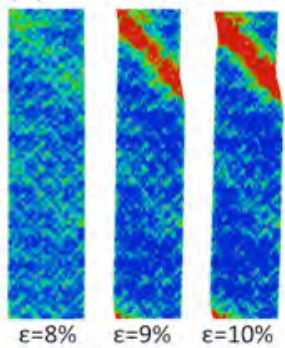

0.6

(e)

\section{$5 \mathrm{~nm}$}

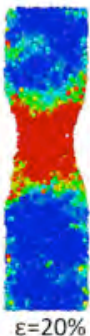

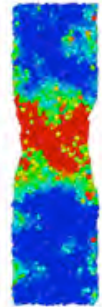

$\varepsilon=15 \%$

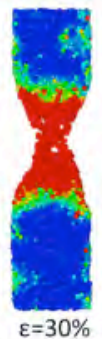

$\varepsilon^{\mathrm{vM}}$

Figure 9. Molecular dynamics simulations on the effect of sample size (i.e. nanopillar diameter) on the mechanical behaviour of the $\mathrm{Zr}$-Ni-Al metallic glass. The height of the nanopillars was selected such that the aspect ratio was constant at a value of 3 for height to diameter. (a) Tensile engineering stress versus strain for samples with diameters denoted in the legend (b-e) Analysis of the atomic von Mises shear strain $\left(\varepsilon^{\mathrm{vM}}\right)$ of the nanopillar samples with the diameters of (b) $40 \mathrm{~nm}$, (c) $20 \mathrm{~nm}$, (d) $10 \mathrm{~nm}$, and (e) $5 \mathrm{~nm}$ indicates a transition from brittle shear banding to ductile necking by decreasing the sample size. 


\section{Graphical Abstract}

\section{As-sputtered $\sim 150 \mathrm{~nm}$ wide specimen}
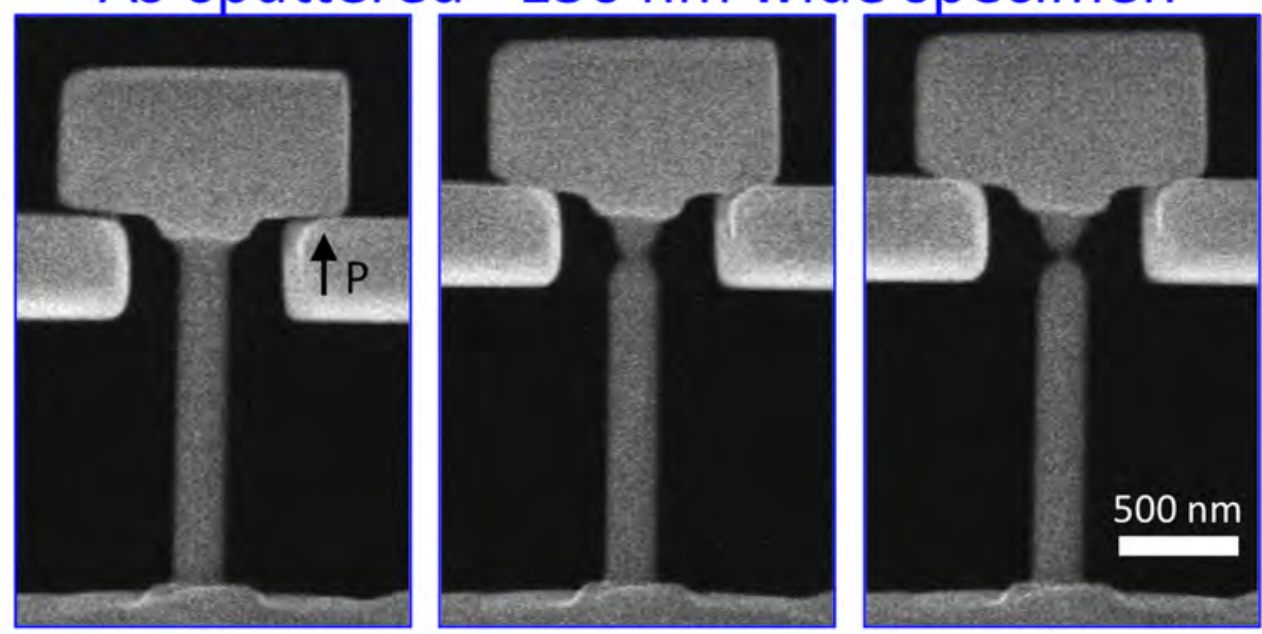

Annealed

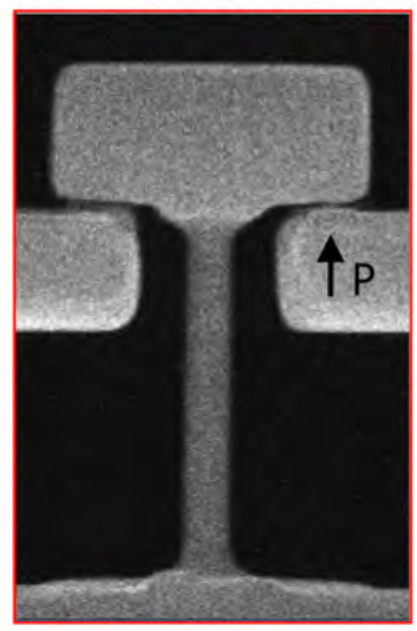

$\sim 150 \mathrm{~nm}$ wide specimen
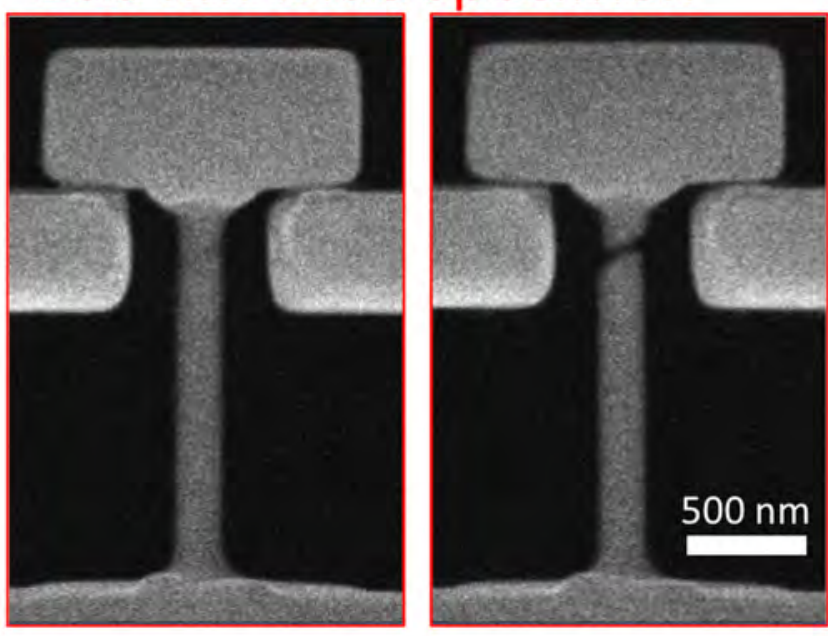